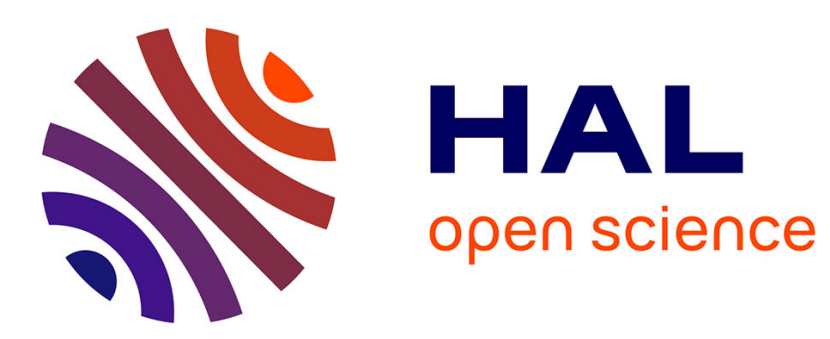

\title{
Walks with small steps in the quarter plane
} Mireille Bousquet-Mélou, Marni Mishna

\section{- To cite this version:}

Mireille Bousquet-Mélou, Marni Mishna. Walks with small steps in the quarter plane. Contemporary mathematics, 2010, 520, pp.1-40. hal-00333741v3

\section{HAL Id: hal-00333741 \\ https://hal.science/hal-00333741v3}

Submitted on 17 Oct 2009

HAL is a multi-disciplinary open access archive for the deposit and dissemination of scientific research documents, whether they are published or not. The documents may come from teaching and research institutions in France or abroad, or from public or private research centers.
L'archive ouverte pluridisciplinaire HAL, est destinée au dépôt et à la diffusion de documents scientifiques de niveau recherche, publiés ou non, émanant des établissements d'enseignement et de recherche français ou étrangers, des laboratoires publics ou privés. 
WALKS WITH SMALL STEPS IN THE QUARTER PLANE

\author{
MIREILLE BOUSQUET-MÉLOU AND MARNI MISHNA
}

\begin{abstract}
Let $\mathcal{S} \subset\{-1,0,1\}^{2} \backslash\{(0,0)\}$. We address the enumeration of plane lattice walks with steps in $\mathcal{S}$, that start from $(0,0)$ and always remain in the first quadrant $\{(i, j): i \geq$ $0, j \geq 0\}$. A priori, there are $2^{8}$ problems of this type, but some are trivial. Some others are equivalent to a model of walks confined to a half-plane: such models can be solved systematically using the kernel method, which leads to algebraic generating functions. We focus on the remaining cases, and show that there are 79 inherently different problems to study.

To each of them, we associate a group $G$ of birational transformations. We show that this group is finite (of order at most 8 ) in 23 cases, and infinite in the 56 other cases.

We present a unified way of solving 22 of the 23 models associated with a finite group. For all of them, the generating function is found to be D-finite. The 23rd model, known as Gessel's walks, has recently been proved by Bostan et al. to have an algebraic (and hence D-finite) solution. We conjecture that the remaining 56 models, associated with an infinite group, have a non-D-finite generating function.

Our approach allows us to recover and refine some known results, and also to obtain new results. For instance, we prove that walks with N, E, W, S, SW and NE steps have an algebraic generating function.
\end{abstract}

\title{
1. Introduction
}

The enumeration of lattice walks is a classical topic in combinatorics. Many combinatorial objects (trees, maps, permutations, lattice polygons, Young tableaux, queues...) can be encoded by lattice walks, so that lattice path enumeration has many applications. Given a lattice, for instance the hypercubic lattice $\mathbb{Z}^{d}$, and a finite set of steps $\mathcal{S} \subset \mathbb{Z}^{d}$, a typical problem is to determine how many $n$-step walks with steps taken from $\mathcal{S}$, starting from the origin, are confined to a certain region $\mathcal{A}$ of the space. If $\mathcal{A}$ is the whole space, then the length generating function of these walks is a simple rational series. If $\mathcal{A}$ is a half-space, bounded by a rational hyperplane, the associated generating function is an algebraic series. Instances of the latter problem have been studied in many articles since at least the end of the 19th century [1, 6]. It is now understood that the kernel method provides a systematic solution to all such problems, which are, in essence, one-dimensional 14, 2]. Other generic approaches to half-space problems are provided in 20, 25].

A natural next class of problems is the enumeration of walks constrained to lie in the intersection of two rational half-spaces - typically, in the quarter plane. Thus far, a number of instances have been solved, but no unified approach has emerged yet, and the problem is far from being completely understood. The generating functions that have been found demonstrate a more complicated structure than those of half-space problems. Some examples are algebraic, but for reasons that are poorly understood combinatorially [26, 13, 40, 9. Some examples are, more generally, D-finite, meaning that the generating function satisfies a linear differential equation with polynomial coefficients [31, 15, 11, 47. Some examples are not D-finite, having infinitely many singularities in the complex plane [15, 41].

We focus in this paper on walks in the plane confined to the first quadrant. The four examples of Figure 1 illustrate the complexity of this problem:

\footnotetext{
MBM was supported by the French "Agence Nationale de la Recherche", project SADA ANR-05-BLAN-0372.
}

MM was supported by a Canadian NSERC Discovery grant. 
- Kreweras' walks (steps W, S, and NE): these were first counted in 1965 by Kreweras 37. He obtained a complicated expression for the number of $n$-step walks ending at $(i, j)$, which simplifies drastically when $j=0$. It was then proved by Gessel that the associated 3-variable generating function is algebraic [26]. Since then, simpler derivations of this series have been obtained [42, 13], including an automated proof [36], and

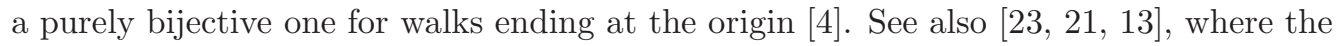
stationary distribution of a related Markov chain in the quarter plane is obtained, and found to be algebraic.

- Gessel's walks (steps E, W, NE and SW): Around 2001, Gessel conjectured a simple hypergeometric formula for the number of $n$-step walks ending at the origin. This conjecture was proved recently by Kauers, Koutschan and Zeilberger [35. Even more recently, Bostan and Kauers proved that the associated 3-variable generating function (which counts all quarter plane walks by the length and the coordinates of the endpoint) is in fact algebraic [9]. Strangely enough, the simple numbers conjectured by Gessel had not been recognized as the coefficients of an algebraic series before. Both approaches involve, among other tools, heavy computer algebra calculations.

- Gouyou-Beauchamps's walks (steps E, W, NW and SE): Gouyou-Beauchamps discovered in 1986 a simple hypergeometric formula for walks ending on the $x$-axis [29]. We derive in this paper similar expressions for the total number of walks, and for those ending at a prescribed position. The associated series are D-finite, but transcendental. These walks are related to Young tableaux of height at most 4 [30]. An affine deformation transforms them into square lattice walks (with N, S, E and W steps) confined to the wedge $0 \leq j \leq i$. An enumeration of these walks involving the number of visits to the diagonal appears in [32, 44, 43.

- A non-D-finite case is provided by walks with NE, NW and SE steps. Mishna and Rechnitzer established a complicated expression for the generating function of these walks, from which they were able to prove that this series has infinitely many singularities, and thus cannot be D-finite [41].

Observe that we have defined $\mathcal{S}$, the set of steps, as a subset of $\mathbb{Z}^{2}$ but that we often use a more intuitive terminology, referring to $(1,1)$ as a NE step, for instance. We occasionally abuse the coordinate notation and directly write our steps using $x$ 's and $y$ 's, writing for example $x \bar{y}$ for a SE step.

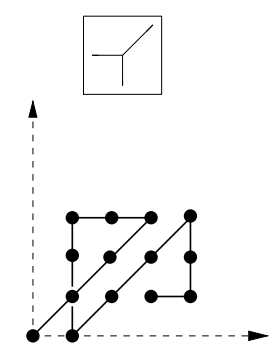

$q(3 m)=\frac{4^{m}(3 m) !}{(m+1) !(2 m+1) !}$

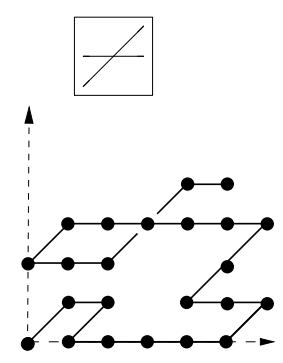

$q(2 m)=16^{m} \frac{(5 / 6)_{m}(1 / 2)_{m}}{(5 / 3)_{m}(2)_{m}}$
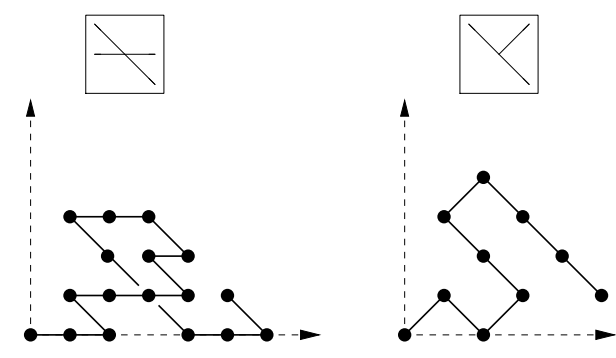

$q(2 m)=\frac{6(2 m) !(2 m+2) !}{m !(m+1) !(m+2) !(m+3) !}$ $q(m)=0$

FiguRE 1. Four models of walks in the quarter plane: Kreweras' walks, Gessel's walks, Gouyou-Beauchamps's walks, and the non-D-finite example of Mishna and Rechnitzer. The numbers $q(n)$ count walks of length $n$ confined to the quarter plane that start and end at the origin. We have used the notation $(a)_{n}=a(a+1) \cdots(a+n-1)$. 
Ideally, we seek generic results or combinatorial conditions which assure D-finite (or even algebraic) generating functions. One criterion of this type states that if the set $\mathcal{S}$ is invariant by reflection around a vertical axis (we say for short that it has a vertical symmetry) and consists of steps $(i, j)$ such that $|i| \leq 1$, then the generating function of quarter plane walks with steps in $\mathcal{S}$ is D-finite 15, 11. This is also true when the set of steps is left invariant by a Weyl group and the walks are confined to a corresponding Weyl chamber [28]. We are not aware of any other such criteria.

\subsection{Results}

We restrict our attention to the quarter plane and small steps (i.e., the step set $\mathcal{S}$ is a subset of $\left.\{-1,0,1\}^{2} \backslash\{(0,0)\}\right)$. This includes the four examples above. We first narrow down the $2^{8}$ possible cases to 79 distinct non-trivial problems (Section 2). Their step sets $\mathcal{S}$ are listed in Tables 1 to 1 in Section 8. These problems fall into two categories, depending on whether a certain group associated with $\mathcal{S}$ is finite or infinite (Section 3). The 23 models associated with a finite group turn out to be those that satisfy at least one of the following properties:

- the step set possesses a vertical symmetry,

- the vector sum of the vectors in the step set is 0 .

In Section 1 we develop certain general tools that apply to all models - in particular, we explain how to write for each of them a functional equation that defines the generating function of the walks. Then, we describe a uniform way to solve this equation for all of the models associated with a finite group, except one (Gessel's walks). The solutions are all D-finite, and even algebraic in three cases (Sections 5 and (6). Note that Gessel's walks are also known to have an algebraic generating function [9]. We conjecture that all the solutions to models with an infinite group are non-D-finite. We conclude in Section 1 with some comments and questions. The tables of Section 8 list the 79 models, classified according to the order of the corresponding group, and provide references to both the existing literature and to the relevant result of this paper.

\subsection{Comments and detailed outline of the paper}

The following technical and/or bibliographical comments may be of interest to readers who have already worked on similar problems. One will also find here a more detailed description of the contents of the paper.

The starting point of our approach is a functional equation defining the generating function $Q(x, y ; t)$ that counts quarter plane walks by the length (variable $t$ ) and the coordinates of the endpoint (variables $x$ and $y$ ). This equation merely reflects a step by step construction of quarter plane walks. For instance, the equation obtained for Kreweras' walks (first example in Figure 1 ) reads:

$$
(1-t(1 / x+1 / y+x y)) Q(x, y ; t)=1-t / x Q(0, y ; t)-t / y Q(x, 0 ; t) .
$$

Note that there is no obvious way to derive from the above identity an equation for, say, $Q(0,0 ; t)$ or $Q(1,1 ; t)$. Following Zeilberger's terminology [56], we say that the variables $x$ and $y$ are catalytic. One of our objectives is to provide some general principles that may be applied to any such linear equation with two catalytic variables. The case of linear equations with one catalytic variable is well-understood, and the solutions are always algebraic [14].

One key tool in our approach is a certain group $G(\mathcal{S})$ of birational transformations that leaves the kernel of the functional equation (that is, the coefficient of $Q(x, y ; t)$ ) unchanged (Section (3). We have borrowed this group from the little yellow book by Fayolle, Iasnogorodski and Malyshev [21, in which the authors study the stationary distributions of Markov chains with small steps in the quarter plane. Ever since it was imported from probability theory to combinatorics, this group has proved useful (in several disguises, like the obstinate, algebraic, or iterated kernel method) to solve various enumeration problems, dealing with walks 11, 13, 1.5, 33, 40, 41, but also with other objects, like permutations [12] or set partitions [17, 55 - the common feature of all these problems being that they boil down to solving a linear equation 
with two catalytic variables. A striking observation, which applies to all solutions obtained so far, is that the solution is D-finite if and only if the group is finite. We find that exactly 23 out of our 79 quarter plane models give rise to a finite group.

We then focus on these 23 models. For each of them, we derive in Section 1 an identity between various specializations of $Q(x, y ; t)$ which we call the orbit sum (or half-orbit sum, when there is an $x / y$ symmetry in $G(\mathcal{S}))$.

In Section 5, we show how to derive $Q(x, y ; t)$ from the orbit sum in 19 out of the 20 models that have a finite group and no $x / y$ symmetry. The number of $n$-step walks ending at $(i, j)$ is obtained by extracting the coefficient of $x^{i} y^{j} t^{n}$ in a rational series which is easily obtained from the step set and the group. This implies that the generating function $Q(x, y ; t)$ is D-finite. The form of the solution is reminiscent of a formula obtained by Gessel and Zeilberger for the enumeration of walks confined to a Weyl chamber, when the set of steps is invariant under the associated Weyl group 28]. Indeed, when the quarter plane problem happens to be a Weyl chamber problem, our method can be seen as an algebraic version of the reflection principle (which is the basis of [28]). However, its range of applications seems to be more general. We work out in details three cases: walks with N, W and SE steps (equivalent to Young tableaux with at most 3 rows), walks with N, S, E, W, SE and NW steps (which do not seem to have been solved before, but behave very much like the former case), and finally walks with E, W, NW and SE steps (studied in 29]), for which we obtain new explicit results.

The results of Section 6 may be considered more surprising: For the 3 models that have a finite group and an $x / y$ symmetry, we derive the series $Q(x, y ; t)$ from the half-orbit sum and find, remarkably, that $Q(x, y ; t)$ is always algebraic. We work out in details all cases: walks with S, W and NE steps (Kreweras' walks), walks with E, N and SW steps (the reverse steps of Kreweras' steps) and walks with N, S, E, W, NE and SW steps, which, to our knowledge, have never been studied before. In particular, we find that the series $Q(1,1 ; t)$ that counts walks of the latter type, regardless of their endpoint, satisfies a simple quartic equation.

\subsection{Preliminaries and notation}

Let $A$ be a commutative ring and $x$ an indeterminate. We denote by $A[x]$ (resp. $A[[x]])$ the ring of polynomials (resp. formal power series) in $x$ with coefficients in $A$. If $A$ is a field, then $A(x)$ denotes the field of rational functions in $x$, and $A((x))$ the field of Laurent series in $x$. These notations are generalized to polynomials, fractions and series in several indeterminates. We denote $\bar{x}=1 / x$, so that $A[x, \bar{x}]$ is the ring of Laurent polynomials in $x$ with coefficients in $A$. The coefficient of $x^{n}$ in a Laurent series $F(x)$ is denoted $\left[x^{n}\right] F(x)$. The valuation of a Laurent series $F(x)$ is the smallest $d$ such that $x^{d}$ occurs in $F(x)$ with a non-zero coefficient.

The main family of series that we use is that of power series in $t$ with coefficients in $A[x, \bar{x}]$, that is, series of the form

$$
F(x ; t)=\sum_{n \geq 0, i \in \mathbb{Z}} f(i ; n) x^{i} t^{n}
$$

where for all $n$, almost all coefficients $f(i ; n)$ are zero. The positive part of $F(x ; t)$ in $x$ is the following series, which has coefficients in $x \mathbb{Q}[x]$ :

$$
\left[x^{>}\right] F(x ; t):=\sum_{n \geq 0, i>0} f(i ; n) x^{i} t^{n} .
$$

We define similarly the negative, non-negative and non-positive parts of $F(x ; t)$ in $x$, which we denote respectively by $\left[x^{<}\right] F(x ; t),\left[x^{\geq}\right] F(x ; t)$ and $[x \leq] F(x ; t)$.

In our generating functions, the indeterminate $t$ keeps track of the length of the walks. We record the coordinates of the endpoints with the variables $x$ and $y$. In order to simplify the notation, we often omit the dependence of our series in $t$, writing for instance $Q(x, y)$ instead of $Q(x, y ; t)$ for the generating function of quarter plane walks.

Recall that a power series $F\left(x_{1}, \ldots, x_{k}\right) \in \mathbb{K}\left[\left[x_{1}, \ldots, x_{k}\right]\right]$, where $\mathbb{K}$ is a field, is algebraic (over $\left.\mathbb{K}\left(x_{1}, \ldots, x_{k}\right)\right)$ if it satisfies a non-trivial polynomial equation $P\left(x_{1}, \ldots, x_{k}, F\left(x_{1}, \ldots, x_{k}\right)\right)=0$. 
It is transcendental if it is not algebraic. It is $D$-finite (or holonomic) if the vector space over $\mathbb{K}\left(x_{1}, \ldots, x_{k}\right)$ spanned by all partial derivatives of $F\left(x_{1}, \ldots, x_{k}\right)$ has finite dimension. This means that for all $i \leq k$, the series $F$ satisfies a (non-trivial) linear differential equation in $x_{i}$ with coefficients in $\mathbb{K}\left[x_{1}, \ldots, x_{k}\right]$. We refer to [38, 39] for a study of these series. All algebraic series are D-finite. In Section 5 we use the following result.

Proposition 1. If $F(x, y ; t)$ is a rational power series in $t$, with coefficients in $\mathbb{C}(x)[y, \bar{y}]$, then $\left[y^{>}\right] F(x, y ; t)$ is algebraic over $\mathbb{C}(x, y, t)$. If the latter series has coefficients in $\mathbb{C}[x, \bar{x}, y]$, its positive part in $x$, that is, the series $\left[x^{>}\right]\left[y^{>}\right] F(x, y ; t)$, is a 3 -variable $D$-finite series (in $x, y$ and $t$ ).

The first statement is a simple adaptation of 25, Thm. 6.1]. The key tool is to expand $F(x, y ; t)$ in partial fractions of $y$. The second statement relies on the fact that the diagonal of a D-finite series is D-finite [38. One first observes that there is some $k$ such that $\left[y^{>}\right] F\left(x, y ; t x^{k}\right)$ has polynomial coefficients in $x$ and $y$, and then applies Remark (4), page 377 of [38].

Below we also use the fact that a series $F(t)$ with real coefficients such that $\left[t^{n}\right] F(t) \sim \kappa \mu^{n} n^{-k}$ with $k \in\{1,2,3, \ldots\}$ cannot be algebraic [22].

\section{The number of non-equivalent non-simple models}

Since we restrict ourselves to walks with "small" steps (sometimes called steps with small variations), there are only a finite number of cases to study, namely $2^{8}$, the number of sets $\mathcal{S}$ formed of small steps. However, some of these models are trivial (for instance $\mathcal{S}=\emptyset$, or $\mathcal{S}=\{\bar{x}\}$ ). More generally, it sometimes happens that one of the two constraints imposed by the quarter plane holds automatically, at least when the other constraint is satisfied. Such models are equivalent to problems of walks confined to a half-space: their generating function is always algebraic and can be derived automatically using the kernel method 14, 2. We show in Section 2.1 that, out of the $2^{8}=256$ models, only 138 are truly 2-constraint problems and are thus worth considering in greater detail. Then, some of the remaining problems coincide up to an $x / y$ symmetry and are thus equivalent. As shown in Section 2.2, one finally obtains 79 inherently different, truly 2-constraint problems.

\subsection{Easy algebraic cases}

Let us say that a step $(i, j)$ is $x$-positive if $i>0$. We define similarly $x$-negative, $y$-positive and $y$-negative steps. There are a number of reasons that may make the enumeration of quarter plane walks with steps in $\mathcal{S}$ a simple problem:

(1) If $\mathcal{S}$ contains no $x$-positive step, we can ignore its $x$-negative steps, which will never be used in a quarter plane walk: we are thus back to counting walks with vertical steps on a (vertical) half-line. The solution of this problem is always algebraic, and even rational if $\mathcal{S}=\emptyset$ or $\mathcal{S}=\{y\}$ or $\mathcal{S}=\{\bar{y}\}$

(2) Symmetrically, if $\mathcal{S}$ contains no $y$-positive step, the problem is simple with an algebraic solution;

(3) If $\mathcal{S}$ contains no $x$-negative step, all walks with steps in $\mathcal{S}$ that start from $(0,0)$ lie in the half-plane $i \geq 0$. Thus any walk lying weakly above the $x$-axis is automatically a quarter plane walk, and the problem boils down to counting walks confined to the upper half-plane: the corresponding generating function is always algebraic;

(4) Symmetrically, if $\mathcal{S}$ contains no $y$-negative step, the problem is simple with an algebraic solution.

We can thus restrict our attention to sets $\mathcal{S}$ containing $x$-positive, $x$-negative, $y$-positive and $y$-negative steps. An inclusion-exclusion argument shows that the number of such sets is 161 . More precisely, the polynomial that counts them by cardinality is

$$
\begin{aligned}
P_{1}(z)=(1+z)^{8}-4(1+z)^{5}+2(1+z)^{2}+ & 4(1+z)^{3}-4(1+z)+1 \\
& =2 z^{2}+20 z^{3}+50 z^{4}+52 z^{5}+28 z^{6}+8 z^{7}+z^{8} .
\end{aligned}
$$


In the expression of $P_{1}(z)$, one of the 4 terms $(1+z)^{5}$ counts sets with no $x$-positive step, one term $(1+z)^{2}$ those with no $x$-positive nor $x$-negative step, one term $(1+z)^{3}$ those with no $x$ positive nor $y$-positive step, and so on. All the sets $\mathcal{S}$ we have discarded correspond to problems that either are trivial or can be solved automatically using the kernel method.

Among the remaining 161 sets $\mathcal{S}$, some do not contain any step with both coordinates nonnegative: in this case the only quarter plane walk is the empty walk. These sets are subsets of $\{\bar{x}, \bar{y}, x \bar{y}, \bar{x} \bar{y}, \bar{x} y\}$. But, as we have assumed at this stage that $\mathcal{S}$ contains $x$-positive and $y$-positive steps, both $x \bar{y}$ and $\bar{x} y$ must belong to $\mathcal{S}$. Hence we exclude $2^{3}$ of our 161 step sets, which leaves us with 153 sets, the generating polynomial of which is

$$
P_{2}(z)=P_{1}(z)-z^{2}(1+z)^{3}=z^{2}+17 z^{3}+47 z^{4}+51 z^{5}+28 z^{6}+8 z^{7}+z^{8} .
$$

Another, slightly less obvious, source of simplicity of the model is when one of the quarter plane constraints implies the other. Assume that all walks with steps in $\mathcal{S}$ that end at a non-negative abscissa automatically end at a non-negative ordinate (we say, for short, that the $x$-condition forces the $y$-condition). This implies in particular that the steps $\bar{y}$ and $x \bar{y}$ do not belong to $\mathcal{S}$. As we have assumed that $\mathcal{S}$ contains a $y$-negative step, $\bar{x} \bar{y}$ must be in $\mathcal{S}$. But then $x$ cannot belong to $\mathcal{S}$, otherwise some walks with a non-negative final abscissa would have a negative final ordinate, like $x$ followed by $\bar{x} \bar{y}$. We are left with sets $\mathcal{S} \subset\{\bar{x}, y, x y, \bar{x} y, \bar{x} \bar{y}\}$ containing $\bar{x} \bar{y}$, and also $x y$ (because we need at least one $x$-positive step). Observe that these five steps are those lying above the first diagonal. Conversely, it is easy to realize that for any such set, the $x$-condition forces the $y$-condition. The generating polynomial of such super-diagonal sets is $z^{2}(1+z)^{3}$. Symmetrically, we need not consider sub-diagonal sets. An inclusion-exclusion argument reduces the generating polynomial of non-simple cases to

$$
P_{3}(z)=P_{2}(z)-2 z^{2}(1+z)^{3}+z^{2}=11 z^{3}+41 z^{4}+49 z^{5}+28 z^{6}+8 z^{7}+z^{8},
$$

that is to say, to 138 sets $\mathcal{S}$.

\subsection{Symmetries}

The eight symmetries of the square act on the step sets. However, only the $x / y$ symmetry (reflection across the first diagonal) leaves the quarter plane fixed. Thus two step sets obtained from one another by applying this symmetry lead to equivalent counting problems. As we want to count non-equivalent problems, we need to determine how many among the 138 sets $\mathcal{S}$ that are left have the $x / y$ symmetry. We repeat the arguments of the previous subsection, counting only symmetric models. We successively obtain

$$
\begin{gathered}
P_{1}^{\mathrm{sym}}=(1+z)^{2}\left(1+z^{2}\right)^{3}-2(1+z)\left(1+z^{2}\right)+1, \\
P_{2}^{\mathrm{sym}}=P_{1}^{\mathrm{sym}}-z^{2}(1+z)\left(1+z^{2}\right), \\
P_{3}^{\mathrm{sym}}=P_{2}^{\mathrm{sym}}-z^{2}=3 z^{3}+5 z^{4}+5 z^{5}+4 z^{6}+2 z^{7}+z^{8} .
\end{gathered}
$$

For instance, the term we subtract from $P_{1}^{\text {sym }}$ to obtain $P_{2}^{\text {sym }}$ counts symmetric subsets of $\{\bar{x}, \bar{y}, x \bar{y}, \bar{x} \bar{y}, \bar{x} y\}$ containing $x \bar{y}$ and $\bar{x} y$. The generating polynomial of (inherently different) models that are neither trivial, nor equivalent to a 1-constraint problem is thus

$$
\frac{1}{2}\left(P_{3}+P_{3}^{\mathrm{sym}}\right)=7 z^{3}+23 z^{4}+27 z^{5}+16 z^{6}+5 z^{7}+z^{8} .
$$

This gives a total of 79 models, shown in Tables 1 to 1 .

\section{The group of the walk}

Let $\mathcal{S}$ be a set of small steps containing $x$-positive, $x$-negative, $y$-positive and $y$-negative steps. This includes the 79 sets we wish to study. Let $S(x, y)$ denote the generating polynomial of the steps of $\mathcal{S}$ :

$$
S(x, y)=\sum_{(i, j) \in \mathcal{S}} x^{i} y^{j}
$$


It is a Laurent polynomial in $x$ and $y$. Recall that $\bar{x}$ stands for $1 / x$, and $\bar{y}$ for $1 / y$. Let us write

$$
S(x, y)=A_{-1}(x) \bar{y}+A_{0}(x)+A_{1}(x) y=B_{-1}(y) \bar{x}+B_{0}(y)+B_{1}(y) x .
$$

By assumption, $A_{1}, B_{1}, A_{-1}$ and $B_{-1}$ are non-zero. Clearly, $S(x, y)$ is left unchanged by the following rational transformations:

$$
\Phi:(x, y) \mapsto\left(\bar{x} \frac{B_{-1}(y)}{B_{1}(y)}, y\right) \quad \text { and } \quad \Psi:(x, y) \mapsto\left(x, \bar{y} \frac{A_{-1}(x)}{A_{1}(x)}\right) .
$$

Note that both $\Phi$ and $\Psi$ are involutions, and thus birational transformations. By composition, they generate a group that we denote $G(\mathcal{S})$, or $G$ if there is no risk of confusion. This group is isomorphic to a dihedral group $D_{n}$ of order $2 n$, with $n \in \mathbb{N} \cup\{\infty\}$. For each $g \in G$, one has $S(g(x, y))=S(x, y)$. The sign of $g$ is 1 (resp. -1) if $g$ is the product of an even (resp. odd) number of generators $\Phi$ and $\Psi$.

\section{Examples}

1. Assume $\mathcal{S}$ is left unchanged by a reflection across a vertical line. This is equivalent to saying that $S(x, y)=S(\bar{x}, y)$, or that $B_{1}(y)=B_{-1}(y)$, or that $A_{i}(x)=A_{i}(\bar{x})$ for $i=-1,0,1$. Then the orbit of $(x, y)$ under the action of $G$ reads

$$
(x, y) \stackrel{\Phi}{\longrightarrow}(\bar{x}, y) \stackrel{\Psi}{\longrightarrow}(\bar{x}, C(x) \bar{y}) \stackrel{\Phi}{\longrightarrow}(x, C(x) \bar{y}) \stackrel{\Psi}{\longrightarrow}(x, y),
$$

with $C(x)=\frac{A_{-1}(x)}{A_{1}(x)}$, so that $G$ is finite of order 4 .

Note that there may exist rational transformations on $(x, y)$ that leave $S(x, y)$ unchanged but are not in $G$. For instance, if $\mathcal{S}=\{\mathrm{N}, \mathrm{S}, \mathrm{E}, \mathrm{W}\}$, the map $(x, y) \mapsto(y, x)$ leaves $S(x, y)$ unchanged, but the orbit of $(x, y)$ under $G$ is $\{(x, y),(\bar{x}, y),(\bar{x}, \bar{y}),(x, \bar{y})\}$.

2. Consider the case $\mathcal{S}=\{\bar{x}, y, x \bar{y}\}$. We have $A_{-1}(x)=x, A_{1}(x)=1, B_{-1}(y)=1, B_{1}(y)=\bar{y}$. The transformations are

$$
\Phi:(x, y) \mapsto(\bar{x} y, y) \quad \text { and } \quad \Psi:(x, y) \mapsto(x, x \bar{y}),
$$

and they generate a group of order 6 :

$$
(x, y) \stackrel{\Phi}{\longrightarrow}(\bar{x} y, y) \stackrel{\Psi}{\longrightarrow}(\bar{x} y, \bar{x}) \stackrel{\Phi}{\longrightarrow}(\bar{y}, \bar{x}) \stackrel{\Psi}{\longrightarrow}(\bar{y}, x \bar{y}) \stackrel{\Phi}{\longrightarrow}(x, x \bar{y}) \stackrel{\Psi}{\longrightarrow}(x, y) .
$$

3. Consider now the case $\mathcal{S}=\{\bar{x}, \bar{y}, x y\}$, which differs from the previous one by a rotation of 90 degrees. We have $A_{-1}(x)=1, A_{1}(x)=x, B_{-1}(y)=1, B_{1}(y)=y$. The two transformations are

$$
\Phi:(x, y) \mapsto(\bar{x} \bar{y}, y) \quad \text { and } \quad \Psi:(x, y) \mapsto(x, \bar{x} \bar{y})
$$

and they also generate a group of order 6 :

$$
(x, y) \stackrel{\Phi}{\longrightarrow}(\bar{x} \bar{y}, y) \stackrel{\Psi}{\longrightarrow}(\bar{x} \bar{y}, x) \stackrel{\Phi}{\longrightarrow}(y, x) \stackrel{\Psi}{\longrightarrow}(y, \bar{x} \bar{y}) \stackrel{\Phi}{\longrightarrow}(x, \bar{x} \bar{y}) \stackrel{\Psi}{\longrightarrow}(x, y) .
$$

As shown by the following lemma, this is not a coincidence.

Lemma 2. Let $\mathcal{S}$ and $\tilde{\mathcal{S}}$ be two sets of steps differing by one of the 8 symmetries of the square. Then the groups $G(\mathcal{S})$ and $G(\tilde{\mathcal{S}})$ are isomorphic.

Proof. The group of symmetries of the square is generated by the two reflections $\Delta$ (across the first diagonal) and $V$ (across a vertical line). Hence it suffices to prove the lemma when $\tilde{S}=\Delta(S)$ and when $\tilde{S}=V(S)$. We denote by $\Phi$ and $\Psi$ the transformations associated with $\mathcal{S}$, and by $\tilde{\Phi}$ and $\tilde{\Psi}$ those associated with $\tilde{\mathcal{S}}$.

Assume $\tilde{S}=\Delta(S)$. We have $\tilde{A}_{i}(x)=B_{i}(x)$ and $\tilde{B}_{i}(y)=A_{i}(y)$. Denote by $\delta$ the involution that swaps the coordinates of a pair: $\delta(x, y)=(y, x)$. An elementary calculation gives

$$
\tilde{\Phi}=\delta \circ \Psi \circ \delta \quad \text { and } \quad \tilde{\Psi}=\delta \circ \Phi \circ \delta
$$

so that the groups $G(\mathcal{S})$ and $G(\tilde{\mathcal{S}})$ are conjugate by $\delta$. 
Assume now $\tilde{S}=V(S)$. We have $\tilde{A}_{i}(x)=A_{i}(\bar{x})$ and $\tilde{B}_{i}(y)=B_{-i}(y)$. Denote by $v$ the involution that replaces the first coordinate of a pair by its reciprocal: $v(x, y)=(\bar{x}, y)$. An elementary calculation gives

$$
\tilde{\Phi}=v \circ \Phi \circ v \quad \text { and } \quad \tilde{\Psi}=v \circ \Psi \circ v
$$

so that the groups $G(\mathcal{S})$ and $G(\tilde{\mathcal{S}})$ are conjugate by $v$.

Theorem 3. Out of the 79 models under consideration, exactly 23 are associated with a finite group:

- 16 have a vertical symmetry and thus a group of order 4 ,

- 5 have a group of order 6 ,

- 2 have a group of order 8 .

Proof. Given that $\Phi$ and $\Psi$ are involutions, the group they generate is finite of order $2 n$ if and only if $\Theta:=\Psi \circ \Phi$ has finite order $n$. It is thus easy to prove that one of the groups $G(\mathcal{S})$ has order $2 n$ : one computes the $m^{\text {th }}$ iterate $\Theta^{m}$ for $1 \leq m \leq n$, and checks that only the last of these transformations is the identity.

We have already seen in the examples above that models with a vertical symmetry have a group of order 4 . We leave it to the reader to check that the models of Tables 2 and 3 have groups of order 6 and 8 , respectively. These tables give the orbit of $(x, y)$ under the action of $G$, the elements being listed in the following order: $(x, y), \Phi(x, y), \Psi \circ \Phi(x, y)$, and so on.

Proving that one of the groups $G(\mathcal{S})$ is infinite is a more difficult task. We apply two different strategies, depending on $\mathcal{S}$. The first one uses valuations and works for the five step sets of Figure 2. These are the sets of our collection for which all elements $(i, j)$ satisfy $i+j \geq 0$. We are very grateful to Jason Bell, who suggested to us a second strategy which turned out to apply to the remaining cases.
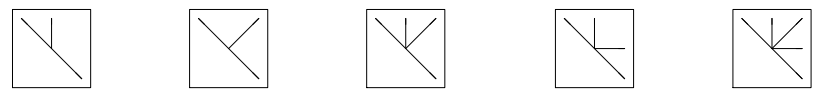

FiguRE 2. Five step sets with an infinite group.

\section{The valuation argument}

Let $z$ be an indeterminate, and let $x$ and $y$ be Laurent series in $z$ with coefficients in $\mathbb{Q}$, of respective valuations $a$ and $b$. We assume that the trailing coefficients of these series, namely $\left[z^{a}\right] x$ and $\left[z^{b}\right] y$, are positive. Let us define $x^{\prime}$ by $\Phi(x, y)=\left(x^{\prime}, y\right)$. Then the trailing coefficient of $x^{\prime}$ (and $y$ ) is positive, and the valuation of $x^{\prime}$ (and $y$ ) only depends on $a$ and $b$ :

$$
\phi(a, b):=\left(\operatorname{val}\left(x^{\prime}\right), \operatorname{val}(y)\right)= \begin{cases}\left(-a+b\left(v_{-1}^{(y)}-v_{1}^{(y)}\right), b\right) & \text { if } b \geq 0 \\ \left(-a+b\left(d_{-1}^{(y)}-d_{1}^{(y)}\right), b\right) & \text { if } b \leq 0\end{cases}
$$

where $v_{i}^{(y)}$ (resp. $d_{i}^{(y)}$ ) denotes the valuation (resp. degree) in $y$ of $B_{i}(y)$, for $i= \pm 1$. Similarly, $\Psi(x, y):=\left(x, y^{\prime}\right)$ is well-defined, and the valuations of $x$ and $y^{\prime}$ only depend on $a$ and $b$ :

$$
\psi(a, b):=\left(\operatorname{val}(x), \operatorname{val}\left(y^{\prime}\right)\right)= \begin{cases}\left(a,-b+a\left(v_{-1}^{(x)}-v_{1}^{(x)}\right)\right) & \text { if } a \geq 0 \\ \left(a,-b+a\left(d_{-1}^{(x)}-d_{1}^{(x)}\right)\right) & \text { if } a \leq 0\end{cases}
$$

where $v_{i}^{(x)}$ (resp. $d_{i}^{(x)}$ ) denotes the valuation (resp. degree) in $x$ of $A_{i}(x)$, for $i= \pm 1$.

In order to prove that $G$ is infinite, it suffices to prove that the group $G^{\prime}$ generated by $\phi$ and $\psi$ is infinite. To prove the latter statement, it suffices to exhibit $(a, b) \in \mathbb{Z}^{2}$ such that the orbit of $(a, b)$ under the action of $G^{\prime}$ is infinite. 
Let $\mathcal{S}$ be one of the five sets of Figure 2. Then $A_{-1}(x)=x$ and $B_{-1}(y)=y$, so that $v_{-1}^{(x)}=d_{-1}^{(x)}=v_{-1}^{(y)}=d_{-1}^{(y)}=1$. Also, $v_{1}^{(x)}=v_{1}^{(y)}=-1$ as $\mathcal{S}$ contains the steps $\bar{x} y$ and $x \bar{y}$. Hence the transformations $\phi$ and $\psi$ read:

$\phi(a, b)=\left\{\begin{array}{ll}(-a+2 b, b) & \text { if } b \geq 0, \\ \left(-a+b\left(1-d_{1}^{(y)}\right), b\right) & \text { if } b \leq 0,\end{array} \quad \psi(a, b)= \begin{cases}(a, 2 a-b) & \text { if } a \geq 0 \\ \left(a,-b+a\left(1-d_{1}^{(x)}\right)\right) & \text { if } a \leq 0 .\end{cases}\right.$

It is easy to check, by induction on $n \geq 0$, that

$$
(\psi \circ \phi)^{n}(1,2)=(2 n+1,2 n+2) \quad \text { and } \quad \phi(\psi \circ \phi)^{n}(1,2)=(2 n+3,2 n+2) .
$$

(All these pairs have positive entries, so that we never need to know $d_{1}^{(y)}$ or $d_{1}^{(x)}$.) Hence the orbit of $(1,2)$ under the action of $\phi$ and $\psi$ is infinite, and so are the groups $G^{\prime}$ and $G$.

We believe, from our computer experiments, that the groups $G^{\prime}$ associated with the remaining 51 models of Table 1 are finite, and hence, cannot be used to prove that $G$ is infinite. Instead, we use for these models a different argument based on the fixed points of $\Theta=\Psi \circ \Phi$.

\section{The fixed point argument}

We are left with 51 models. Thanks to Lemma 2, we only need to prove that (roughly) a quarter of them are associated with a finite group: if $G(\mathcal{S})$ is infinite, then $G(\tilde{\mathcal{S}})$ is infinite for all sets $\tilde{\mathcal{S}}$ that differ from $\mathcal{S}$ by a symmetry of the square $]$. This leaves 14 models to study, listed in Table 5.

Assume $\Theta=\Psi \circ \Phi$ is well-defined in the neighborhood of $(a, b) \in \mathbb{C}^{2}$, and that this point is fixed by $\Theta$. Note that $a$ and $b$ are algebraic over $\mathbb{Q}$. Let us write $\Theta=\left(\Theta_{1}, \Theta_{2}\right)$, where $\Theta_{1}$ and $\Theta_{2}$ are the two coordinates of $\Theta$. Each $\Theta_{i}$ sends the pair $(x, y)$ to a rational function of $x$ and $y$. The local expansion of $\Theta$ around $(a, b)$ reads

$$
\Theta(a+u, b+v)=(a, b)+(u, v) J_{a, b}+O\left(u^{2}\right)+O\left(v^{2}\right)+O(u v),
$$

where $J_{a, b}$ is the Jacobian matrix of $\Theta$ at $(a, b)$ :

$$
J_{a, b}=\left(\begin{array}{ll}
\frac{\partial \Theta_{1}}{\partial x}(a, b) & \frac{\partial \Theta_{2}}{\partial x}(a, b) \\
\frac{\partial \Theta_{1}}{\partial y}(a, b) & \frac{\partial \Theta_{2}}{\partial y}(a, b)
\end{array}\right) .
$$

Iterating the above expansion gives, for $m \geq 1$,

$$
\Theta^{m}(a+u, b+v)=(a, b)+(u, v) J_{a, b}^{m}+O\left(u^{2}\right)+O\left(v^{2}\right)+O(u v) .
$$

Assume $G(\mathcal{S})$ is finite of order $2 n$, so that $\Theta$ has order $n$. Then $\Theta^{n}(a+u, b+v)=(a, b)+(u, v)$, and the above equation shows that $J_{a, b}^{n}$ is the identity matrix. In particular, all eigenvalues of $J_{a, b}$ are roots of unity.

This gives us a strategy for proving that a group $G(\mathcal{S})$ is infinite: find a fixed point $(a, b)$ for $\Theta$, and compute the characteristic polynomial $\chi(X)$ of the Jacobian matrix $J_{a, b}$. This is a polynomial in $X$ with coefficients in $\mathbb{Q}(a, b)$. In order to decide whether the roots of $\chi$ are roots of unity, we eliminate $a$ and $b$ (which are algebraic numbers) from the equation $\chi(X)=0$ to obtain a polynomial $\bar{\chi}(X) \in \mathbb{Q}[X]$ that vanishes for all eigenvalues of $J_{a, b}$ : if none of its factors is cyclotomic, we can conclude that $G(\mathcal{S})$ is infinite. As all cyclotomic polynomials of given degree are known, this procedure is effective.

Let us treat one case in detail, say $\mathcal{S}=\{x, y, \bar{y}, \bar{x} \bar{y}\}$ (the first case in Table 5 ). We have

$$
\Theta(x, y)=\Psi \circ \Phi(x, y)=(\bar{x} \bar{y}, x+\bar{y}) .
$$

\footnotetext{
${ }^{1}$ Why a quarter, rather than an eighth? Recall that, if two (distinct) models differ by an $x / y$ symmetry, only one of them appears in Table
} 
Every pair $(a, b)$ such that $a^{4}+a^{3}=1$ and $b=1 / a^{2}$ is fixed by $\Theta$. Let us choose one such pair. The Jacobian matrix reads

$$
J_{a, b}=\left(\begin{array}{cc}
-1 & 1 \\
-a^{3} & -a^{4}
\end{array}\right),
$$

and its characteristic polynomial is

$$
\chi(X):=\operatorname{det}\left(X \operatorname{Id}-J_{a, b}\right)=X^{2}+X\left(1+a^{4}\right)+a^{3}+a^{4} .
$$

Let $X$ be a root of this polynomial. By eliminating $a$ (which satisfies $a^{4}+a^{3}=1$ ), we obtain

$$
\bar{\chi}(X):=X^{8}+9 X^{7}+31 X^{6}+62 X^{5}+77 X^{4}+62 X^{3}+31 X^{2}+9 X+1=0 .
$$

This polynomial is irreducible, and distinct from all cyclotomic polynomials of degree 8 . Hence none of its roots are roots of unity, no power of $J_{a, b}$ is equal to the identity matrix, and the $\operatorname{group} G(\mathcal{S})$ is infinite.

This strategy turns out to work for all models of Table 5 . This table gives, for each model, the algebraic equations defining the fixed point $(a, b)$ that we choose (for instance, the "condition" $a^{4}+a^{3}-1$ occurring on the first line means that $a^{4}+a^{3}-1=0$ ), and a polynomial $\bar{\chi}(X) \in \mathbb{Q}[X]$ that vanishes at all eigenvalues of the Jacobian matrix, in factored form. One then checks that no factor of this polynomial is cyclotomic.

It may be worth noting that this second strategy does not work for the five models of Figure 2: in the first three cases, $\Theta$ has no fixed point; in the last two cases, it has a fixed point $(a, b)$, but the sixth power of the Jacobian matrix $J_{a, b}$ is the identity (of course, $\Theta^{6}$ is not the identity; more precisely, the expansion of $\Theta^{6}(a+u, b+v)$ involves cubic terms in $u$ and $\left.v\right)$.

Remark. To put this discussion in a larger framework, let us mention that the group of birational transformations or $\mathbb{C}^{2}$ (or of the projective plane $\mathbb{P}^{2}(\mathbb{C})$ ), called the Cremona group, has been the object of many studies in algebraic geometry since the end of the 19th century [34, 54]. It seems possible that, given the attention already paid to the classification of finite subgroups of

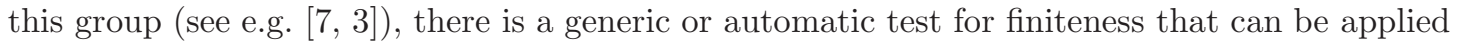
to all our examples.

\section{General tools}

Let $\mathcal{S}$ be one of the 79 step sets of Tables 1 to . Let $\mathcal{Q}$ be the set of walks that start from $(0,0)$, take their steps from $\mathcal{S}$ and always remain in the first quadrant. Let $q(i, j ; n)$ be the number of such walks that have length $n$ and end at position $(i, j)$. Denote by $Q(x, y ; t) \equiv Q(x, y)$ the associated generating function:

$$
Q(x, y ; t)=\sum_{i, j, n \geq 0} q(i, j ; n) x^{i} y^{j} t^{n} .
$$

It is a formal power series in $t$ with coefficients in $\mathbb{Q}[x, y]$.

\subsection{A functional equation}

Lemma 4. As a power series in $t$, the generating function $Q(x, y) \equiv Q(x, y ; t)$ of walks with steps taken from $\mathcal{S}$ starting from $(0,0)$ and staying in the first quadrant is characterized by the following functional equation:

$$
K(x, y) x y Q(x, y)=x y-t x A_{-1}(x) Q(x, 0)-t y B_{-1}(y) Q(0, y)+t \epsilon Q(0,0)
$$

where

$$
K(x, y)=1-t S(x, y)=1-t \sum_{(i, j) \in \mathcal{S}} x^{i} y^{j}
$$

is called the kernel of the equation, the polynomials $A_{-1}(x)$ and $B_{-1}(y)$ are the coefficients of $\bar{y}$ and $\bar{x}$ in $S(x, y)$, as described by (2), and $\epsilon$ is 1 if $(-1,-1)$ is one of the allowed steps, and 0 otherwise. 
Proof. We construct walks step by step, starting from the empty walk and concatenating a new step at the end of the walk at each stage. The empty walk has weight 1 . The generating function of walks obtained by adding a step of $\mathcal{S}$ at the end of a walk of $\mathcal{Q}$ is $t S(x, y) Q(x, y)$. However, some of these walks exit the quadrant: those obtained by concatenating a $y$-negative step to a walk ending at ordinate 0 , and those obtained by concatenating an $x$-negative step to a walk ending at abscissa 0 . Walks ending at ordinate (resp. abscissa) 0 are counted by the series $Q(x, 0)$ (resp. $Q(0, y))$. Hence we must subtract the series $t \bar{y} A_{-1}(x) Q(x, 0)$ and $t \bar{x} B_{-1}(y) Q(0, y)$. However, if $(-1,-1) \in \mathcal{S}$, we have subtracted twice the series counting walks obtained by concatenating this step to a walk ending at $(0,0)$ : we must thus add the series $\epsilon t \bar{x} \bar{y} Q(0,0)$. This inclusion-exclusion argument gives

$$
Q(x, y)=1+t S(x, y) Q(x, y)-t \bar{y} A_{-1}(x) Q(x, 0)-t \bar{x} B_{-1}(y) Q(0, y)+\epsilon t \bar{x} \bar{y} Q(0,0),
$$

which, multiplied by $x y$, gives the equation of the lemma.

The fact that it characterizes $Q(x, y ; t)$ completely (as a power series in $t$ ) comes from the fact that the coefficient of $t^{n}$ in $Q(x, y ; t)$ can be computed inductively using this equation. This is of course closely related to the fact that we have used a recursive description of walks in $\mathcal{Q}$ to obtain the equation.

\subsection{Orbit sums}

We have seen in Section 3 that all transformations $g$ of the group $G$ associated with the step set $\mathcal{S}$ leave the polynomial $S(x, y)$ unchanged. Hence they also leave the kernel $K(x, y)=1-t S(x, y)$ unchanged. Write the equation of Lemma 4 as

$$
K(x, y) x y Q(x, y)=x y-F(x)-G(y)+t \epsilon Q(0,0),
$$

with $F(x)=t x A_{-1}(x) Q(x, 0)$ and $G(y)=t y B_{-1}(y) Q(0, y)$. Replacing $(x, y)$ by $\Phi(x, y)=\left(x^{\prime}, y\right)$ gives

$$
K(x, y) x^{\prime} y Q\left(x^{\prime}, y\right)=x^{\prime} y-F\left(x^{\prime}\right)-G(y)+t \epsilon Q(0,0) .
$$

The difference between the former and latter identities reads:

$$
K(x, y)\left(x y Q(x, y)-x^{\prime} y Q\left(x^{\prime}, y\right)\right)=x y-x^{\prime} y-F(x)+F\left(x^{\prime}\right) .
$$

The term $G(y)$ has disappeared. We can repeat this process, and add to this identity the functional equation of Lemma 4 , evaluated at $\left(x^{\prime}, y^{\prime}\right)=\Psi\left(x^{\prime}, y\right)$. This gives:

$$
K(x, y)\left(x y Q(x, y)-x^{\prime} y Q\left(x^{\prime}, y\right)+x^{\prime} y^{\prime} Q\left(x^{\prime}, y^{\prime}\right)\right)=x y-x^{\prime} y+x^{\prime} y^{\prime}-F(x)-G\left(y^{\prime}\right)+t \epsilon Q(0,0) \text {. }
$$

Now the term $F\left(x^{\prime}\right)$ has disappeared. If $G$ is finite of order $2 n$, we can repeat the procedure until we come back to $(\Psi \circ \Phi)^{n}(x, y)=(x, y)$. That is to say, we form the alternating sum of the equations over the orbit of $(x, y)$. All unknown functions on the right-hand side finally vanish, giving the following proposition, where we use the notation

$$
\text { for } g \in G, \quad g(A(x, y)):=A(g(x, y)) \text {. }
$$

Proposition 5 (Orbit sums). Assume the group $G(\mathcal{S})$ is finite. Then

$$
\sum_{g \in G} \operatorname{sign}(g) g(x y Q(x, y ; t))=\frac{1}{K(x, y ; t)} \sum_{g \in G} \operatorname{sign}(g) g(x y) .
$$

Observe that the right-hand side is a rational function in $x, y$ and $t$. We show in Section 5 that this identity implies immediately that 19 of the 23 models having a finite group have a D-finite solution.

The 4 remaining models are Gessel's model $\{x, \bar{x}, x y, \bar{x} \bar{y}\}$ (which we do not solve in this paper) and the three models with steps $\mathcal{S}_{1}=\{\bar{x}, \bar{y}, x y\}, \mathcal{S}_{2}=\{x, y, \bar{x} \bar{y}\}$, and $\mathcal{S}=\mathcal{S}_{1} \cup \mathcal{S}_{2}$. For each of these three models, the orbit of $(x, y)$ is

$$
(x, y) \stackrel{\Phi}{\longrightarrow}(\bar{x} \bar{y}, y) \stackrel{\Psi}{\longrightarrow}(\bar{x} \bar{y}, x) \stackrel{\Phi}{\longrightarrow}(y, x) \stackrel{\Psi}{\longrightarrow}(y, \bar{x} \bar{y}) \stackrel{\Phi}{\longrightarrow}(x, \bar{x} \bar{y}) \stackrel{\Psi}{\longrightarrow}(x, y),
$$


and exhibits an $x / y$ symmetry. That is, $(y, x)$ belongs to the orbit of $(x, y)$. Moreover, if $g((x, y))=(y, x)$, then $\operatorname{sign}(g)=-1$. Thus the right-hand side of (4) vanishes, leaving

$$
x y Q(x, y)-\bar{x} Q(\bar{x} \bar{y}, y)+\bar{y} Q(\bar{x} \bar{y}, x)=x y Q(y, x)-\bar{x} Q(y, \bar{x} \bar{y})+\bar{y} Q(x, \bar{x} \bar{y}) .
$$

But this identity directly follows from the obvious relation $Q(x, y)=Q(y, x)$ and does not bring much information. In Section 6 , we solve these three obstinate models by summing the functional equation over one half of the orbit only. Given that $A_{-1}(x)=B_{-1}(x)$, the identity resulting from this half-orbit summation reads as follows.

Proposition 6 (Half-orbit sums). Denote $\mathcal{S}_{1}=\{\bar{x}, \bar{y}, x y\}$ and $\mathcal{S}_{2}=\{x, y, \bar{x} \bar{y}\}$. If the set of steps is $\mathcal{S}_{1}, \mathcal{S}_{2}$ or $\mathcal{S}_{1} \cup \mathcal{S}_{2}$, then

$$
x y Q(x, y)-\bar{x} Q(\bar{x} \bar{y}, y)+\bar{y} Q(\bar{x} \bar{y}, x)=\frac{x y-\bar{x}+\bar{y}-2 t x A_{-1}(x) Q(x, 0)+t \epsilon Q(0,0)}{K(x, y)} .
$$

Remark. For Gessel's walks, the orbit of $(x, y)$ is shown in Table 3. Proposition 5 reads

$$
\sum_{g \in G} \operatorname{sign}(g) g(x y Q(x, y))=0,
$$

although no obvious symmetry explains this identity.

\subsection{The roots of the kernel}

Recall that the kernel of the main functional equation (Lemma 4 ) is

$$
K(x, y)=1-t \sum_{(i, j) \in \mathcal{S}} x^{i} y^{j} .
$$

Lemma 7. Let

$$
\Delta(x)=\left(1-t A_{0}(x)\right)^{2}-4 t^{2} A_{-1}(x) A_{1}(x) .
$$

Let $Y_{0}(x)$ and $Y_{1}(x)$ denote the two roots of the kernel $K(x, y)$, where $K(x, y)$ is seen as a polynomial in $y$. These roots are Laurent series in $t$ with coefficients in $\mathbb{Q}(x)$ :

$$
Y_{0}(x)=\frac{1-t A_{0}(x)-\sqrt{\Delta(x)}}{2 t A_{1}(x)}, \quad Y_{1}(x)=\frac{1-t A_{0}(x)+\sqrt{\Delta(x)}}{2 t A_{1}(x)} .
$$

Their valuations in $t$ are respectively 1 and -1 . Moreover, $1 / K(x, y)$ is a power series in $t$ with coefficients in $\mathbb{Q}[x, \bar{x}, y, \bar{y}]$, and the coefficient of $y^{j}$ in this series can be easily extracted using

$$
\frac{1}{K(x, y)}=\frac{1}{\sqrt{\Delta(x)}}\left(\frac{1}{1-\bar{y} Y_{0}(x)}+\frac{1}{1-y / Y_{1}(x)}-1\right) .
$$

Proof. The equation $K(x, Y)=0$ also reads

$$
Y=t\left(A_{-1}(x)+Y A_{0}(x)+Y^{2} A_{1}(x)\right) .
$$

Solving this quadratic provides the expressions of $Y_{0}(x)$ and $Y_{1}(x)$ given above. As $\Delta(x)=$ $1+O(t)$, the series $Y_{1}$ has valuation -1 in $t$, and first term $1 /\left(t A_{1}(x)\right)$. The equation

$$
Y_{0}(x) Y_{1}(x)=\frac{A_{-1}(x)}{A_{1}(x)}
$$

then shows that $Y_{0}(x)$ has valuation 1 . This is also easily seen on (7), which in turn implies that $Y_{0}$ has coefficients in $\mathbb{Q}[x, \bar{x}]$. The equation

$$
Y_{0}(x)+Y_{1}(x)=\frac{1}{t A_{1}(x)}-\frac{A_{0}(x)}{A_{1}(x)}
$$

shows that for $n \geq 1$, the coefficient of $t^{n}$ in $Y_{1}(x)$ is also a Laurent polynomial in $x$. This is not true, in general, of the coefficients of $t^{-1}$ and $t^{0}$. 
The identity (6) results from a partial fraction expansion in $y$. Note that both $Y_{0}$ and $1 / Y_{1}$ have valuation 1 in $t$. Hence the expansion in $y$ of $1 / K(x, y)$ gives

$$
\left[y^{j}\right] \frac{1}{K(x, y)}= \begin{cases}\frac{Y_{0}(x)^{-j}}{\sqrt{\Delta(x)}} & \text { if } j \leq 0 \\ \frac{Y_{1}(x)^{-j}}{\sqrt{\Delta(x)}} & \text { if } j \geq 0 .\end{cases}
$$

\subsection{Canonical factorization of the discriminant $\Delta(x)$}

The kernel can be seen as a polynomial in $y$. Its discriminant is then a (Laurent) polynomial in $x$ :

$$
\Delta(x)=\left(1-t A_{0}(x)\right)^{2}-4 t^{2} A_{-1}(x) A_{1}(x) .
$$

Say $\Delta$ has valuation $-\delta$, and degree $d$ in $x$. Then it admits $\delta+d$ roots $X_{i} \equiv X_{i}(t)$, for $1 \leq i \leq \delta+d$, which are Puiseux series in $t$ with complex coefficients. Exactly $\delta$ of them, say $X_{1}, \ldots, X_{\delta}$, are finite (and actually vanish) at $t=0$. The remaining $d \operatorname{roots}, X_{\delta+1}, \ldots, X_{\delta+d}$, have a negative valuation in $t$ and thus diverge at $t=0$. (We refer to [52, Chapter 6] for generalities on solutions of algebraic equations with coefficients in $\mathbb{C}(t)$.) We write

$$
\Delta(x)=\Delta_{0} \Delta_{-}(\bar{x}) \Delta_{+}(x),
$$

with

$$
\begin{aligned}
& \Delta_{-}(\bar{x}) \equiv \Delta_{-}(\bar{x} ; t)=\prod_{i=1}^{\delta}\left(1-\bar{x} X_{i}\right) \\
& \Delta_{+}(x) \equiv \Delta_{+}(x ; t)=\prod_{i=\delta+1}^{\delta+d}\left(1-x / X_{i}\right)
\end{aligned}
$$

and

$$
\Delta_{0} \equiv \Delta_{0}(t)=(-1)^{\delta} \frac{\left[\bar{x}^{\delta}\right] \Delta(x)}{\prod_{i=1}^{\delta} X_{i}}=(-1)^{d}\left[x^{d}\right] \Delta(x) \prod_{i=\delta+1}^{\delta+d} X_{i} .
$$

It can be seen that $\Delta_{0}$ (resp. $\left.\Delta_{-}(\bar{x}), \Delta_{+}(x)\right)$ is a formal power series in $t$ with constant term 1 and coefficients in $\mathbb{C}$ (resp. $\mathbb{C}[\bar{x}], \mathbb{C}[x]$ ). The above factorization is an instance of the canonical factorization of series in $\mathbb{Q}[x, \bar{x}][[t]]$, which was introduced by Gessel [25], and has proved useful in several walk problems since then 10, 16, 13. It will play a crucial role in Section 6 .

\section{D-finite solutions via orbit sums}

In this section, we first show that 19 of the 23 models having a finite group can be solved from the corresponding orbit sum. This includes the 16 models having a vertical symmetry, plus 3 others. Then, we work out the latter 3 models in details, obtaining closed form expressions for the number of walks ending at prescribed positions.

\subsection{A general result}

Proposition 8. For the 23 models associated with a finite group, except from the four cases $\mathcal{S}=\{\bar{x}, \bar{y}, x y\}, \mathcal{S}=\{x, y, \bar{x} \bar{y}\}, \mathcal{S}=\{x, y, \bar{x}, \bar{y}, x y, \bar{x} \bar{y}\}$ and $\mathcal{S}=\{x, \bar{x}, x y, \bar{x} \bar{y}\}$, the following holds. The rational function

$$
R(x, y ; t)=\frac{1}{K(x, y ; t)} \sum_{g \in G} \operatorname{sign}(g) g(x y)
$$

is a power series in $t$ with coefficients in $\mathbb{Q}(x)[y, \bar{y}]$ (Laurent polynomials in $y$, having coefficients in $\mathbb{Q}(x))$. Moreover, the positive part in $y$ of $R(x, y ; t)$, denoted $R^{+}(x, y ; t)$, is a power series in 
$t$ with coefficients in $\mathbb{Q}[x, \bar{x}, y]$. Extracting the positive part in $x$ of $R^{+}(x, y ; t)$ gives $x y Q(x, y ; t)$. In brief,

$$
x y Q(x, y ; t)=\left[x^{>}\right]\left[y^{>}\right] R(x, y ; t) .
$$

In particular, $Q(x, y ; t)$ is $D$-finite. The number of $n$-step walks ending at $(i, j)$ is

$$
q(i, j ; n)=\left[x^{i+1} y^{j+1}\right]\left(\sum_{g \in G} \operatorname{sign}(g) g(x y)\right) S(x, y)^{n}
$$

where

$$
S(x, y)=\sum_{(p, q) \in \mathcal{S}} x^{p} y^{q} .
$$

Proof. We begin with the 16 models associated with a group of order 4 (Table 1). We will then address the three remaining cases, $\mathcal{S}=\{\bar{x}, y, x \bar{y}\}, \mathcal{S}=\{x, \bar{x}, x \bar{y}, \bar{x} y\}$ and $\mathcal{S}=\{x, \bar{x}, y, \bar{y}, x \bar{y}, \bar{x} y\}$.

All models with a group of order 4 exhibit a vertical symmetry. That is, $K(x, y)=K(\bar{x}, y)$. As discussed in the examples of Section 3 , the orbit of $(x, y)$ reads

$$
(x, y) \stackrel{\Phi}{\longleftrightarrow}(\bar{x}, y) \stackrel{\Psi}{\longleftrightarrow}(\bar{x}, C(x) \bar{y}) \stackrel{\Phi}{\longleftrightarrow}(x, C(x) \bar{y}) \stackrel{\Psi}{\longleftrightarrow}(x, y),
$$

with $C(x)=\frac{A_{-1}(x)}{A_{1}(x)}$. The orbit sum of Proposition 0 reads

$$
x y Q(x, y)-\bar{x} y Q(\bar{x}, y)+\bar{x} \bar{y} C(x) Q(\bar{x}, C(x) \bar{y})+x \bar{y} C(x) Q(x, C(x) \bar{y})=R(x, y) .
$$

Clearly, both sides of this identity are series in $t$ with coefficients in $\mathbb{Q}(x)[y, \bar{y}]$. Let us extract the positive part in $y$ : only the first two terms of the left-hand side contribute, and we obtain

$$
x y Q(x, y)-\bar{x} y Q(\bar{x}, y)=R^{+}(x, y) .
$$

It is now clear from the left-hand side that $R^{+}(x, y)$ has coefficients in $\mathbb{Q}[x, \bar{x}, y]$. Extracting the positive part in $x$ gives the expression (9) for $x y Q(x, y)$, since the second term of the left-hand side does not contribute.

Let us now examine the cases $\mathcal{S}=\{\bar{x}, y, x \bar{y}\}, \mathcal{S}=\{x, \bar{x}, x \bar{y}, \bar{x} y\}$ and $\mathcal{S}=\{x, \bar{x}, y, \bar{y}, x \bar{y}, \bar{x} y\}$. For each of them, the orbit of $(x, y)$ consists of pairs of the form $\left(x^{a} y^{b}, x^{c} y^{d}\right)$ for integers $a, b, c$ and $d$ (see Tables 2 and 3). This implies that $R(x, y)$ is a series in $t$ with coefficients in $\mathbb{Q}[x, \bar{x}, y, \bar{y}]$. When extracting the positive part in $x$ and $y$ from the orbit sum of Proposition 5 , it is easily checked, in each of the three cases, that only the term $x y Q(x, y)$ remains in the left-hand side. The expression of $x y Q(x, y)$ follows.

Proposition 1 then implies that $Q(x, y ; t)$ is D-finite. The expression of $q(i, j ; n)$ follows from a mere coefficient extraction.

\subsection{Two models with algebraic specializations: $\{\bar{x}, y, x \bar{y}\}$ and $\{x, \bar{x}, y, \bar{y}, x \bar{y}, \bar{x} y\}$}

Consider the case $\mathcal{S}=\{\bar{x}, y, x \bar{y}\} \equiv\{\mathrm{W}, \mathrm{N}, \mathrm{SE}\}$. A walk $w$ with steps taken from $\mathcal{S}$ remains in the first quadrant if each of its prefixes contains more $\mathrm{N}$ steps that SE steps, and more SE steps than W steps. These walks are thus in bijection with Young tableaux of height at most 3 (Figure 3), or, via the Schensted correspondence [4], with involutions having no decreasing subsequence of length 4 . The enumeration of Young tableaux is a well-understood topic. In particular, the number of tableaux of a given shape - and hence the number of $n$-step walks ending at a prescribed position - can be written in closed form using the hook-length formula 49]. It is also known that the total number of tableaux of size $n$ and height at most 3 is the $n$th Motzkin number [48]. Here, we recover these two results (and refine the latter), using orbit and half-orbit sums. Then, we show that the case $\mathcal{S}=\{x, \bar{x}, y, \bar{y}, x \bar{y}, \bar{x} y\}$, which, to our knowledge, has never been solved, behaves very similarly. In particular, the total number of $n$-step walks confined to the quadrant is also related to Motzkin numbers (Proposition 10). 


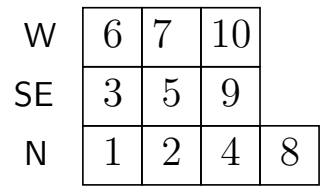

\section{N-N-SE-N-SE-W-W-N-SE-W}

Figure 3. A Young tableau of height 3 and the corresponding quarter plane walk with steps in $\{\mathrm{W}, \mathrm{N}, \mathrm{SE}\}$.

Proposition 9. The generating function of walks with steps W, N, SE confined to the quarter plane is the non-negative part (in $x$ and $y$ ) of a rational series in $t$ having coefficients in $\mathbb{Q}[x, \bar{x}, y, \bar{y}]$ :

$$
Q(x, y ; t)=\left[x^{\geq} y^{\geq}\right] \tilde{R}(x, y ; t), \quad \text { with } \quad \tilde{R}(x, y ; t)=\frac{(1-\bar{x} \bar{y})\left(1-\bar{x}^{2} y\right)\left(1-x \bar{y}^{2}\right)}{1-t(\bar{x}+y+x \bar{y})} .
$$

In particular, $Q(x, y)$ is D-finite. The number of walks of length $n=3 m+2 i+j$ ending at $(i, j)$ is

In particular,

$$
q(i, j ; n)=\frac{(i+1)(j+1)(i+j+2)(3 m+2 i+j) !}{m !(m+i+1) !(m+i+j+2) !}
$$

$$
q(0,0 ; 3 m)=\frac{2(3 m) !}{m !(m+1) !(m+2) !} \sim \sqrt{3} \frac{3^{3 m}}{\pi m^{4}},
$$

so that $Q(0,0 ; t)$ - and hence $Q(x, y ; t)$ - is transcendental.

However, the specialization $Q(x, 1 / x ; t)$ is algebraic of degree 2 :

$$
Q(x, 1 / x ; t)=\frac{1-t \bar{x}-\sqrt{1-2 \bar{x} t+t^{2} \bar{x}^{2}-4 t^{2} x}}{2 x t^{2}} .
$$

In particular, the total number of n-step walks confined to the quadrant is the $n^{\text {th }}$ Motzkin number:

$$
Q(1,1 ; t)=\frac{1-t-\sqrt{(1+t)(1-3 t)}}{2 t^{2}}=\sum_{n \geq 0} t^{n} \sum_{k=0}^{\lfloor n / 2\rfloor} \frac{1}{k+1}\left(\begin{array}{c}
n \\
2 k
\end{array}\right)\left(\begin{array}{c}
2 k \\
k
\end{array}\right) .
$$

Proof. The orbit of $(x, y)$ under the action of $G$ is shown in (3). The first result of the proposition is a direct application of Proposition 8 , with $\tilde{R}(x, y)=R(x, y) /(x y)$. It is then an easy task to extract the coefficient of $x^{i} y^{j} t^{n}$ in $\tilde{R}(x, y ; t)$, using

if $n=3 m+2 i+j$.

$$
\left[x^{i} y^{j}\right](\bar{x}+y+x \bar{y})^{n}=\frac{(3 m+2 i+j) !}{m !(m+i) !(m+i+j) !}
$$

The algebraicity of $Q(x, \bar{x})$ can be proved as follows: let us form the alternating sum of the three equations obtained from Lemma 1 by replacing $(x, y)$ by the first 3 elements of the orbit. These elements are those in which $y$ occurs with a non-negative exponent. This gives

$$
K(x, y)\left(x y Q(x, y)-\bar{x} y^{2} Q(\bar{x} y, y)+\bar{x}^{2} y Q(\bar{x} y, \bar{x})\right)=x y-\bar{x} y^{2}+\bar{x}^{2} y-t x^{2} Q(x, 0)-t \bar{x} Q(0, \bar{x}) .
$$

We now specialize this equation to two values of $y$. First, replace $y$ by $\bar{x}$ : the second and third occurrence of $Q$ in the left-hand side cancel out, leaving

$$
K(x, \bar{x}) Q(x, \bar{x})=1-t x^{2} Q(x, 0)-t \bar{x} Q(0, \bar{x}) .
$$

For the second specialization, replace $y$ by the root $Y_{0}(x)$ of the kernel (see (5)). This is a well-defined substitution, as $Y_{0}(x)$ has valuation 1 in $t$. The left-hand side vanishes, leaving

$$
0=x Y_{0}(x)-\bar{x} Y_{0}(x)^{2}+\bar{x}^{2} Y_{0}(x)-t x^{2} Q(x, 0)-t \bar{x} Q(0, \bar{x}) .
$$


By combining the last two equations, we obtain

$$
K(x, \bar{x}) Q(x, \bar{x})=1-x Y_{0}(x)+\bar{x} Y_{0}(x)^{2}-\bar{x}^{2} Y_{0}(x) .
$$

The expression of $Q(x, \bar{x})$ follows.

The case $\mathcal{S}=\{\mathrm{N}, \mathrm{S}, \mathrm{W}, \mathrm{E}, \mathrm{SE}, \mathrm{NW}\}$ is very similar to the previous one. In particular, the orbit of $(x, y)$ is the same in both cases. The proof of the previous proposition translates almost verbatim. Remarkably, Motzkin numbers occur again.

Proposition 10. The generating function of walks with steps N, S, W, E, SE, NW confined to the quarter plane is the non-negative part (in $x$ and $y$ ) of a rational function:

$$
Q(x, y ; t)=\left[x^{\geq} y^{\geq}\right] \tilde{R}(x, y ; t), \quad \text { with } \quad \tilde{R}(x, y ; t)=\frac{(1-\bar{x} \bar{y})\left(1-\bar{x}^{2} y\right)\left(1-x \bar{y}^{2}\right)}{1-t(x+y+\bar{x}+\bar{y}+x \bar{y}+\bar{x} y)} .
$$

In particular, $Q(x, y ; t)$ is D-finite. The specialization $Q(x, 1 / x ; t)$ is algebraic of degree 2 :

$$
Q(x, 1 / x ; t)=\frac{1-t x-t \bar{x}+\sqrt{(1-t(x+\bar{x}))^{2}-4 t^{2}(1+x)(1+\bar{x})}}{2 t^{2}(1+x)(1+\bar{x})} .
$$

In particular, the total number of $n$-step walks confined to the quadrant is $2^{n}$ times the $n^{\text {th }}$ Motzkin number:

$$
Q(1,1 ; t)=\frac{1-2 t-\sqrt{(1+2 t)(1-6 t)}}{8 t^{2}}=\sum_{n \geq 0} t^{n} \sum_{k=0}^{\lfloor n / 2\rfloor} \frac{2^{n}}{k+1}\left(\begin{array}{c}
n \\
2 k
\end{array}\right)\left(\begin{array}{c}
2 k \\
k
\end{array}\right) .
$$

\subsection{The case $\mathcal{S}=\{x, \bar{x}, x \bar{y}, \bar{x} y\}$}

Consider quadrant walks made of E, W, NW and SE steps. These walks are easily seen to be in bijection with pairs of non-intersecting prefixes of Dyck paths: To pass from such a pair to a quadrant walk, parse the pair of paths from left to right and assign a direction (E, W, NW or SE) to each pair of steps, as described in Figure 1 . Hence the number of walks ending at a prescribed position is given by a 2-by-2 Gessel-Viennot determinant 27] and we can expect a closed form expression for this number.
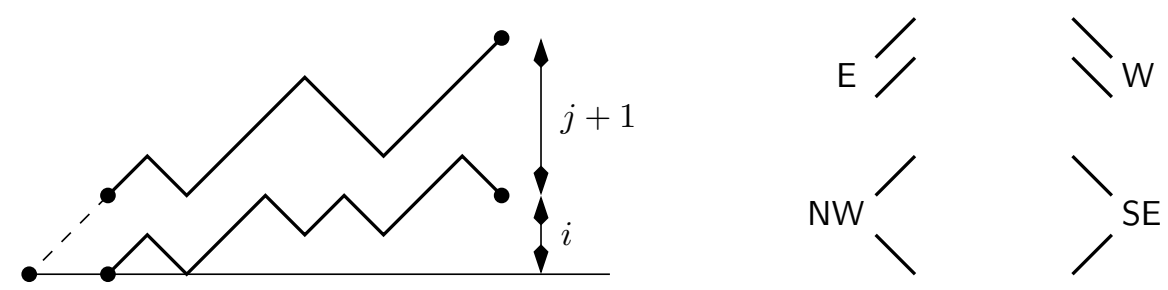

Figure 4. A pair of non-intersecting prefixes of Dyck paths corresponding to the quarter plane walk E-W-E-E-NW-SE-W-E-E-NW.

Also, note that the linear transformation $(i, j) \mapsto(i+j, j)$ maps these quarter plane walks bijectively to walks with $\mathrm{E}, \mathrm{W}, \mathrm{N}$ and $\mathrm{S}$ steps confined to $\{(i, j): 0 \leq j \leq i\}$. In this form, they were studied by Gouyou-Beauchamps, who proved that the number of $n$-step walks ending on the $x$-axis is a product of Catalan numbers [29]. His interest in these walks came from a bijection he had established between walks ending on the $x$-axis and Young tableaux of height at most 4 [30. This bijection is far from being as obvious as the one that relates tableaux of height at most 3 to quarter plane walks with W, N and SW steps (Section 5.2).

Here, we first specialize Proposition 8 to obtain the number of $n$-step walks ending at $(i, j)$ (Proposition 11). Then, we perform an indefinite summation on $i$, or $j$, or both $i$ and $j$ to obtain additional closed form expressions, including Gouyou-Beauchamps's (Corollary 12). 
Proposition 11. The generating function of walks with steps $\mathrm{E}, \mathrm{W}, \mathrm{NW}, \mathrm{SE}$ confined to the quarter plane is the non-negative part (in $x$ and $y$ ) of a rational function:

$$
Q(x, y ; t)=\left[x^{\geq} y^{\geq}\right] \tilde{R}(x, y ; t)
$$

with

$$
\tilde{R}(x, y ; t)=\frac{(1-\bar{x})(1+\bar{x})(1-\bar{y})\left(1-\bar{x}^{2} y\right)(1-x \bar{y})(1+x \bar{y})}{1-t(x+\bar{x}+x \bar{y}+\bar{x} y)} .
$$

In particular, $Q(x, y ; t)$ is $D$-finite. The number of walks of length $n=2 m+i$ ending at $(i, j)$ is

$$
q(i, j ; 2 m+i)=\frac{(i+1)(j+1)(i+j+2)(i+2 j+3)}{(2 m+i+1)(2 m+i+2)(2 m+i+3)^{2}}\left(\begin{array}{c}
2 m+i+3 \\
m-j
\end{array}\right)\left(\begin{array}{c}
2 m+i+3 \\
m+1
\end{array}\right) .
$$

In particular,

$$
q(0,0 ; 2 m)=\frac{6(2 m) !(2 m+2) !}{m !(m+1) !(m+2) !(m+3) !} \sim \frac{24 \cdot 4^{2 m}}{\pi m^{5}}
$$

so that $Q(0,0 ; t)$ - and hence $Q(x, y ; t)$ - is transcendental.

Before we prove this proposition, let us perform summations on $i$ and $j$. Recall that a hypergeometric sequence $(f(k))_{k}$ is Gosper summable (in $k$ ) if there exists another hypergeometric sequence $(g(k))_{k}$ such that $f(k)=g(k+1)-g(k)$. In this case, indefinite summation can be performed in closed form 46, Chapter 5]:

$$
\sum_{k=k_{0}}^{k_{1}} f(k)=g\left(k_{1}+1\right)-g\left(k_{0}\right) \text {. }
$$

The numbers $q(i, j ; n)$ of Proposition 11 have remarkable Gosper properties, from which we now derive Gouyou-Beauchamps's result for walks ending on the $x$-axis, and more.

Corollary 12. The numbers $q(i, j ; n)$ are Gosper-summable in $i$ and in $j$. Hence sums of the form $\sum_{i=i_{0}}^{i_{1}} q(i, j ; n)$ and $\sum_{j=j_{0}}^{j_{1}} q(i, j ; n)$ have closed form expressions. In particular, the number of walks of length $n$ ending at ordinate $j$ is

$$
q(-, j ; n):=\sum_{i \geq 0} q(i, j ; n)= \begin{cases}\frac{(j+1)(2 m) !(2 m+2) !}{(m-j) !(m+1) !^{2}(m+j+2) !} & \text { if } n=2 m, \\ \frac{2(j+1)(2 m+1) !(2 m+2) !}{(m-j) !(m+1) !(m+2) !(m+j+2) !} & \text { if } n=2 m+1 .\end{cases}
$$

Similarly, the number of walks of length $n=2 m+i$ ending at abscissa $i$ is

$$
q(i,-; 2 m+i):=\sum_{j \geq 0} q(i, j ; 2 m+i)=\frac{(i+1)(2 m+i) !(2 m+i+2) !}{m !(m+1) !(m+i+1) !(m+i+2) !} .
$$

In particular, as many $2 m$-step walks end on the $x$ - and $y$-axes:

$$
q(-, 0 ; 2 m)=q(0,-; 2 m)=\frac{(2 m) !(2 m+2) !}{m !(m+1) !^{2}(m+2) !} .
$$

The numbers $q(i,-; n)$ and $q(-, j ; n)$ defined above are again Gosper-summable in $i$ and $j$ respectively. Hence sums of the form

$$
\sum_{i=i_{0}}^{i_{1}} q(i,-; n)=\sum_{i=i_{0}}^{i_{1}} \sum_{j} q(i, j ; n) \quad \text { and } \quad \sum_{j=j_{0}}^{j_{1}} q(-, j ; n)=\sum_{j=j_{0}}^{j_{1}} \sum_{i} q(i, j ; n),
$$


which count walks ending between certain vertical or horizontal lines, have simple closed form expressions. In particular, the total number of quarter plane walks of length $n$ is

$$
q(-,-; n):=\sum_{i, j \geq 0} q(i, j ; n)= \begin{cases}\frac{(2 m) !(2 m+1) !}{m !^{2}(m+1) !^{2}} & \text { if } n=2 m, \\ \frac{(2 m+1) !(2 m+2) !}{m !(m+1) !^{2}(m+2) !} & \text { if } n=2 m+1 .\end{cases}
$$

The asymptotic behaviours of these numbers are found to be

$$
q(-,-; n) \sim c_{1} \cdot 4^{n} / n^{2}, \quad q(0,-; n) \sim c_{2} \cdot 4^{n} / n^{3}, \quad q(-, 0 ; n) \sim c_{3} \cdot 4^{n} / n^{3},
$$

which shows that the series $Q(1,1 ; t), Q(0,1 ; t)$ and $Q(1,0 ; t)$ are transcendental.

Proof of Proposition 11 and Corollary 12. The orbit of $(x, y)$ under the action of $G$, of cardinality 8 , is shown in Table 3. The expression of $Q(x, y ; t)$ given in Proposition 11 is a direct application of Proposition 8, with $\tilde{R}(x, y)=R(x, y) /(x y)$. It is then an easy task to extract the coefficient of $x^{i} y^{j} t^{n}$ in $\tilde{R}(x, y ; t)$, using

$$
x+\bar{x}+\bar{x} y+x \bar{y}=(1+\bar{y})(x+\bar{x} y) \quad \text { and } \quad\left[x^{i} y^{j}\right](x+\bar{x}+\bar{x} y+x \bar{y})^{n}=\left(\begin{array}{c}
n \\
m+i
\end{array}\right)\left(\begin{array}{c}
n \\
m-j
\end{array}\right)
$$

for $n=2 m+i$. This proves Proposition 11 .

For the first part of corollary, that is, the expressions of $q(-, j ; n)$ and $q(i,-; n)$, it suffices to check the following identities, which we have obtained using the implementation of Gosper's algorithm found in the sumtools package of MAPLE:

$$
\begin{aligned}
q(2 i, j ; 2 m) & =g_{1}(i, j ; m)-g_{1}(i+1, j ; m), \\
q(2 i+1, j ; 2 m+1) & =g_{2}(i, j ; m)-g_{2}(i+1, j ; m), \\
q(i, j ; 2 m+i) & =g_{3}(i, j ; m)-g_{3}(i, j+1 ; m),
\end{aligned}
$$

with

$$
\begin{aligned}
g_{1}(i, j ; m) & =\frac{2(1+j)(m+1+2 i(i+1+j))(2 m) !(2 m+1) !}{(m-i-j) !(m-i+1) !(m+i+1) !(m+i+j+2) !}, \\
g_{2}(i, j ; m) & =\frac{2(1+j)\left(m+1+j(1+2 i)+2(1+i)^{2}\right)(2 m+1) !(2 m+2) !}{(m-i-j) !(m-i+1) !(m+i+2) !(m+i+j+3) !}, \\
g_{3}(i, j ; m) & =\frac{(1+i)(m+1+(i+j+1)(1+j))(2 m+i) !(2 m+i+2) !}{(m-j) !(m+1) !(m+i+2) !(m+i+j+2) !} .
\end{aligned}
$$

These three identities respectively lead to

$$
q(-, j ; 2 m)=g_{1}(0, j ; m), \quad q(-, j ; 2 m+1)=g_{2}(0, j ; m) \quad \text { and } \quad q(i,-; 2 m+i)=g_{3}(i, 0 ; m)
$$

as stated in the corollary.

For the second part, we have used the following identities, also discovered (and proved) using MAPle:

$$
\begin{aligned}
q(2 i,-; 2 m) & =g_{4}(i ; m)-g_{4}(i+1 ; m), \\
q(2 i+1,-; 2 m+1) & =g_{5}(i ; m)-g_{5}(i+1 ; m), \\
q(-, j ; 2 m) & =g_{6}(j ; m)-g_{6}(j+1 ; m), \\
q(-, j ; 2 m+1) & =g_{7}(j ; m)-g_{7}(j+1 ; m),
\end{aligned}
$$


with

$$
\begin{aligned}
g_{4}(i ; m) & =\frac{(2 m) !(2 m+1) !}{(m-i) !(m-i+1) !(m+i) !(m+i+1) !}, \\
g_{5}(i ; m) & =\frac{(2 m+1) !(2 m+2) !}{(m-i) !(m-i+1) !(m+i+1) !(m+i+2) !}, \\
g_{6}(j ; m) & =\frac{(2 m) !(2 m+1) !}{(m-j) ! m !(m+1) !(m+j+1) !}, \\
g_{7}(j ; m) & =\frac{(2 m+1) !(2 m+2) !}{(m-j) !(m+1) !(m+2) !(m+j+1) !} .
\end{aligned}
$$

Note that these identities give two ways to determine the total number of $n$-step walks in the quadrant, as

$$
q(-,-; 2 m)=g_{4}(0 ; m)=g_{6}(0 ; m) \quad \text { and } \quad q(-,-; 2 m+1)=g_{5}(0 ; m)=g_{7}(0 ; m) .
$$

\section{Algebraic solutions via half-orbit sums}

In this section we solve in a unified manner the three models whose orbit has an $x / y$ symmetry: $\mathcal{S}_{1}=\{\bar{x}, \bar{y}, x y\}, \mathcal{S}_{2}=\{x, y, \bar{x} \bar{y}\}$ and $\mathcal{S}=\mathcal{S}_{1} \cup \mathcal{S}_{2}$. Remarkably, in all three cases the generating function $Q(x, y ; t)$ is found to be algebraic. Our approach uses the algebraic kernel method introduced by the first author to solve the case $\mathcal{S}=\mathcal{S}_{1}$, that is, Kreweras' model [13, Section 2.3]. We refer to the introduction for more references on this model. The case $\mathcal{S}=\mathcal{S}_{2}$ was solved by the second author in [40], and the case $\mathcal{S}=\mathcal{S}_{1} \cup \mathcal{S}_{2}$ is, to our knowledge, new.

Recall from Proposition 6 that for each of these three models,

$$
x y Q(x, y)-\bar{x} Q(\bar{x} \bar{y}, y)+\bar{y} Q(\bar{x} \bar{y}, x)=\frac{x y-\bar{x}+\bar{y}-2 t x A_{-1}(x) Q(x, 0)+t \epsilon Q(0,0)}{K(x, y)} .
$$

Extract from this equation the coefficient of $y^{0}$ : in the left-hand side, only the second term contributes, and its contribution is $\bar{x} Q_{d}(\bar{x})$, where $Q_{d}(x) \equiv Q_{d}(x ; t)$ is the generating function of walks ending on the diagonal:

$$
Q_{d}(x ; t)=\sum_{n, i \geq 0} t^{n} x^{i} q(i, i ; n) .
$$

The coefficient of $y^{0}$ in the right-hand side can be easily extracted using (8). This gives

$$
-\bar{x} Q_{d}(\bar{x})=\frac{1}{\sqrt{\Delta(x)}}\left(x Y_{0}(x)-\bar{x}+\frac{1}{Y_{1}(x)}-2 t x A_{-1}(x) Q(x, 0)+t \epsilon Q(0,0)\right),
$$

or, given the expression (5) of $Y_{0}$ and the fact that $Y_{0} Y_{1}=\bar{x}$,

$$
\frac{x}{t A_{1}(x)}-\bar{x} Q_{d}(\bar{x})=\frac{1}{\sqrt{\Delta(x)}}\left(\frac{x\left(1-t A_{0}(x)\right)}{t A_{1}(x)}-\bar{x}-2 t x A_{-1}(x) Q(x, 0)+t \epsilon Q(0,0)\right) .
$$

Let us write the canonical factorization of $\Delta(x)=\Delta_{0} \Delta_{+}(x) \Delta_{-}(\bar{x})$ (see Section 4.4). Multiplying the equation by $A_{1}(x) \sqrt{\Delta_{-}(\bar{x})}$ gives

$$
\begin{aligned}
& \sqrt{\Delta_{-}(\bar{x})}\left(\frac{x}{t}-\bar{x} A_{1}(x) Q_{d}(\bar{x})\right)= \\
& \frac{1}{\sqrt{\Delta_{0} \Delta_{+}(x)}}\left(\frac{x\left(1-t A_{0}(x)\right)}{t}-\bar{x} A_{1}(x)-2 t x A_{-1}(x) A_{1}(x) Q(x, 0)+t \epsilon A_{1}(x) Q(0,0)\right) .
\end{aligned}
$$

Each term in this equation is a Laurent series in $t$ with coefficients in $\mathbb{Q}[x, \bar{x}]$. Moreover, very few positive powers of $x$ occur in the left-hand side, while very few negative powers in $x$ occur in the right-hand side. We will extract from this equation the positive and negative parts in $x$, 
and this will give algebraic expressions for the unknown series $Q_{d}(x)$ and $Q(x, 0)$. From now on, we consider each model separately.

\subsection{The case $\mathcal{S}=\{\bar{x}, \bar{y}, x y\}$}

We have $A_{-1}(x)=1, A_{0}(x)=\bar{x}, A_{1}(x)=x$ and $\epsilon=0$. The discriminant $\Delta(x)$ reads $(1-t \bar{x})^{2}-4 t^{2} x$. The curve $\Delta(x ; t)=0$ has a rational parametrization in terms of the series $W \equiv W(t)$, defined as the only power series in $t$ satisfying

$$
W=t\left(2+W^{3}\right) .
$$

Replacing $t$ by $W /\left(2+W^{3}\right)$ in $\Delta(x)$ gives the canonical factorization as $\Delta(x)=\Delta_{0} \Delta_{+}(x) \Delta_{-}(\bar{x})$ with

$$
\Delta_{0}=\frac{4 t^{2}}{W^{2}}, \quad \Delta_{+}(x)=1-x W^{2}, \quad \Delta_{-}(\bar{x})=1-\frac{W\left(W^{3}+4\right)}{4 x}+\frac{W^{2}}{4 x^{2}} .
$$

Extracting the positive part in $x$ from (11) gives

$$
\frac{x}{t}=-\frac{\left(2 t^{2} x^{2} Q(x, 0)-x+2 t\right) W}{2 t^{2} \sqrt{1-x W^{2}}}+\frac{W}{t},
$$

from which we obtain an expression of $Q(x, 0)$ in terms of $W$. Extracting the non-positive part in $x$ from (11) gives

$$
\sqrt{1-\frac{W\left(W^{3}+4\right)}{4 x}+\frac{W^{2}}{4 x^{2}}}\left(\frac{x}{t}-Q_{d}(\bar{x})\right)-\frac{x}{t}=-\frac{W}{t},
$$

from which we obtain an expression of $Q_{d}(\bar{x})$. We recover the results of 123 .

Proposition 13. Let $W \equiv W(t)$ be the power series in $t$ defined by (12). Then the generating function of quarter plane walks formed of $\mathrm{W}, \mathrm{S}$ and $\mathrm{NE}$ steps, and ending on the $x$-axis is

$$
Q(x, 0 ; t)=\frac{1}{t x}\left(\frac{1}{2 t}-\frac{1}{x}-\left(\frac{1}{W}-\frac{1}{x}\right) \sqrt{1-x W^{2}}\right) .
$$

Consequently, the length generating function of walks ending at $(i, 0)$ is

$$
\left[x^{i}\right] Q(x, 0 ; t)=\frac{W^{2 i+1}}{2.4^{i} t}\left(C_{i}-\frac{C_{i+1} W^{3}}{4}\right),
$$

where $C_{i}=\left(\begin{array}{c}2 i \\ i\end{array}\right) /(i+1)$ is the $i$-th Catalan number. The Lagrange inversion formula gives the number of such walks of length $m=3 m+2 i$ as

$$
q(i, 0 ; 3 m+2 i)=\frac{4^{m}(2 i+1)}{(m+i+1)(2 m+2 i+1)}\left(\begin{array}{c}
2 i \\
i
\end{array}\right)\left(\begin{array}{c}
3 m+2 i \\
m
\end{array}\right) .
$$

The generating function of walks ending on the diagonal is

$$
Q_{d}(x ; t)=\frac{W-\bar{x}}{t \sqrt{1-x W\left(1+W^{3} / 4\right)+x^{2} W^{2} / 4}}+\bar{x} / t .
$$

Note that $Q(0,0)$ is algebraic of degree $3, Q(x, 0)$ is algebraic of degree 6 , and $Q(x, y)$ (which can be expressed in terms of $Q(x, 0)$, and $Q(0, y)=Q(y, 0)$ using the functional equation we started from) is algebraic of degree 12 .

\subsection{The case $\mathcal{S}=\{x, y, \bar{x} \bar{y}\}$}

The steps of this model are obtained by reversing the steps of the former model. In particular, the series $Q(0,0)$ counting walks that start and end at the origin is the same in both models. This observation was used in [40] to solve the latter case. We present here a self-contained solution. 
We have $A_{-1}(x)=\bar{x}, A_{0}(x)=x, A_{1}(x)=1$ and $\epsilon=1$. The discriminant $\Delta(x)$ is now $(1-t x)^{2}-4 t^{2} \bar{x}$, and is obtained by replacing $x$ by $\bar{x}$ in the discriminant of the previous model. In particular, the canonical factors of $\Delta(x)$ are

$$
\Delta_{0}=\frac{4 t^{2}}{W^{2}}, \quad \Delta_{+}(x)=1-\frac{W\left(W^{3}+4\right)}{4} x+\frac{W^{2}}{4} x^{2}, \quad \Delta_{-}(\bar{x})=1-\bar{x} W^{2},
$$

where $W \equiv W(t)$ is the power series in $t$ defined by (12). Extracting the coefficient of $x^{0}$ in (11) gives

$$
-\frac{W^{2}}{2 t}=-\frac{W\left(W^{4}+4 W+8 t Q(0,0)\right)}{16 t}
$$

from which we obtain an expression of $Q(0,0)$. Extracting the non-negative part in $x$ from (11) gives

$$
\frac{x}{t}-\frac{W^{2}}{2 t}=-\frac{\left(2 x t^{2} Q(x, 0)-x t^{2} Q(0,0)+t-x^{2}+x^{3} t\right) W}{2 x t^{2} \sqrt{1-x W\left(W^{3}+4\right) / 4+x^{2} W^{2} / 4}}+\frac{W}{2 x t}
$$

from which we obtain an expression of $Q(x, 0)$. Finally, extracting the negative part in $x$ from (11) gives

$$
\left(\frac{x}{t}-\frac{Q_{d}(\bar{x})}{x}\right) \sqrt{1-\frac{W^{2}}{x}}-\frac{x}{t}+\frac{W^{2}}{2 t}=-\frac{W}{2 x t},
$$

from which we obtain an expression of $Q_{d}(\bar{x})$. We have thus recovered, and completed, the results of 40]. Note in particular how simple the number of walks of length $n$ ending at a diagonal point $(i, i)$ is.

Proposition 14. Let $W \equiv W(t)$ be the power series in $t$ defined by (12). Then the generating function of quarter plane walks formed of $\mathrm{N}, \mathrm{E}$ and $\mathrm{SW}$ steps and ending on the $x$-axis is

$Q(x, 0 ; t)=\frac{W\left(4-W^{3}\right)}{16 t}-\frac{t-x^{2}+t x^{3}}{2 x t^{2}}-\frac{\left(2 x^{2}-x W^{2}-W\right) \sqrt{1-x W\left(W^{3}+4\right) / 4+x^{2} W^{2} / 4}}{2 t x W}$.

The generating function of walks ending on the diagonal is

$$
Q_{d}(x ; t)=\frac{x W(x+W)-2}{2 t x^{2} \sqrt{1-x W^{2}}}+\frac{1}{t x^{2}} .
$$

Consequently, the length generating function of walks ending at $(i, i)$ is

$$
\left[x^{i}\right] Q_{d}(x ; t)=\frac{W^{2 i+1}}{4^{i+1} t(i+2)}\left(\begin{array}{c}
2 i \\
i
\end{array}\right)\left(2 i+4-(2 i+1) W^{3}\right) .
$$

The Lagrange inversion formula gives the number of such walks of length $n=3 m+2 i$ as

$$
q(i, i ; 3 m+2 i)=\frac{4^{m}(i+1)^{2}}{(m+i+1)(2 m+2 i+1)}\left(\begin{array}{c}
2 i+1 \\
i
\end{array}\right)\left(\begin{array}{c}
3 m+2 i \\
m
\end{array}\right) .
$$

Note that $Q(0,0)$ is algebraic of degree $3, Q(x, 0)$ is algebraic of degree 6 , and $Q(x, y)$ (which can be expressed in terms of $Q(x, 0), Q(0, y)=Q(y, 0)$ and $Q(0,0)$ using the functional equation we started from) is algebraic of degree 12 .

\subsection{The case $\mathcal{S}=\{x, y, \bar{x}, \bar{y}, x y, \bar{x} \bar{y}\}$}

We have $A_{-1}(x)=1+\bar{x}, A_{0}(x)=x+\bar{x}, A_{1}(x)=1+x$ and $\epsilon=1$. The discriminant $\Delta(x)$ is now $(1-t(x+\bar{x}))^{2}-4 t^{2}(1+x)(1+\bar{x})$, and is symmetric in $x$ and $\bar{x}$. Two of its roots, say $X_{1}$ and $X_{2}$, have valuation 1 in $t$, and the other two roots are $1 / X_{1}$ and $1 / X_{2}$. By studying the two elementary symmetric functions of $X_{1}$ and $X_{2}$ (which are the coefficients of $\Delta_{-}(\bar{x})$ ), we are led to introduce the power series $Z \equiv Z(t)$, satisfying

$$
Z=t \frac{1-2 Z+6 Z^{2}-2 Z^{3}+Z^{4}}{(1-Z)^{2}}
$$


and having no constant term. Replacing $t$ by its expression in terms of $Z$ in $\Delta(x)$ provides the canonical factors of $\Delta(x)$ as

$$
\Delta_{0}=\frac{t^{2}}{Z^{2}}, \quad \Delta_{+}(x)=1-2 Z \frac{1+Z^{2}}{(1-Z)^{2}} x+Z^{2} x^{2}, \quad \Delta_{-}(\bar{x})=\Delta_{+}(\bar{x}) .
$$

As in the previous case, extracting from (11) the coefficient of $x^{0}$ gives and expression of $Q(0,0)$ :

$$
Q(0,0)=\frac{Z\left(1-2 Z-Z^{2}\right)}{t(1-Z)^{2}} .
$$

Extracting then the positive and negative parts of (11) in $x$ gives expressions of $Q(x, 0)$ and $Q_{d}(\bar{x})$.

Proposition 15. Let $Z \equiv Z(t)$ be the power series with no constant term satisfying (13), and denote

$$
\Delta_{+}(x)=1-2 Z \frac{1+Z^{2}}{(1-Z)^{2}} x+Z^{2} x^{2} .
$$

Then the generating function of quarter plane walks formed of N, S, E, W, SE and NW steps, and ending on the $x$-axis is

$$
\begin{aligned}
Q(x, 0 ; t) & =\frac{\left(Z(1-Z)+2 x Z-(1-Z) x^{2}\right) \sqrt{\Delta_{+}(x)}}{2 t x Z(1-Z)(1+x)^{2}} \\
& -\frac{Z(1-Z)^{2}+Z\left(Z^{3}+4 Z^{2}-5 Z+2\right) x-\left(1-2 Z+7 Z^{2}-4 Z^{3}\right) x^{2}+x^{3} Z(1-Z)^{2}}{2 t x Z(1-Z)^{2}(1+x)^{2}} .
\end{aligned}
$$

The generating function of walks ending on the diagonal is

$$
Q_{d}(x ; t)=\frac{1-Z-2 x Z+x^{2} Z(Z-1)}{t x(1+x)(Z-1) \sqrt{\Delta_{+}(x)}}+\frac{1}{t x(1+x)}
$$

Note that $Q(0,0)$ is algebraic of degree $4, Q(x, 0)$ is algebraic of degree 8 , and $Q(x, y)$ (which can be expressed in terms of $Q(x, 0), Q(0, y)=Q(y, 0)$ and $Q(0,0)$ using the functional equation we started from) is algebraic of degree 16 . However, $Q \equiv Q(1,1)$ has degree 4 only, and the algebraic equation it satisfies has a remarkable form:

$$
Q(1+t Q)\left(1+2 t Q+2 t^{2} Q^{2}\right)=\frac{1}{1-6 t}
$$

Also, Motzkin numbers seem to be lurking around, as in Proposition 10.

Corollary 16. Let $N \equiv N(t)$ be the only power series in $t$ satisfying

$$
N=t\left(1+2 N+4 N^{2}\right) .
$$

Up to a factor $t$, this series is the generating function of the numbers $2^{n} M_{n}$, where $M_{n}$ is the $n^{\text {th }}$ Motzkin number:

$$
N=\sum_{n \geq 0} t^{n+1} \sum_{k=0}^{\lfloor n / 2\rfloor} \frac{2^{n}}{k+1}\left(\begin{array}{c}
n \\
2 k
\end{array}\right)\left(\begin{array}{c}
2 k \\
k
\end{array}\right) .
$$

Then the generating function of all walks in the quadrant with steps $N, S, E, W, S E$ and $N W$ is

$$
Q(1,1 ; t)=\frac{1}{2 t}\left(\sqrt{\frac{1+2 N}{1-2 N}}-1\right),
$$

and the generating function of walks in the quadrant ending at the origin is

$$
Q(0,0 ; t)=\frac{(1+4 N)^{3 / 2}}{2 N t}-\frac{1}{2 t^{2}}-\frac{2}{t} .
$$

The proof is elementary once the algebraic equations satisfied by $Q(1,1)$ and $Q(0,0)$ are obtained. 


\section{Final comments and questions}

The above results raise, in our opinion, numerous natural questions. Here are some of them. The first two families of questions are of a purely combinatorial, or even bijective, nature ("explain why some results are so simple"). Others are more closely related to the method used in this paper. We also raise a question of an algorithmic nature.

\subsection{Explain closed form expressions}

We have obtained remarkable hypergeometric expressions for the number of walks in many cases (Propositions 9 to 14). Are there direct combinatorial explanations? Let us underline a few examples that we consider worth investigating.

- Kreweras' walks and their reverse: The number of Kreweras' walks ending at $(i, 0)$ is remarkably simple (Proposition 13). A combinatorial explanation has been found when $i=0$, in connection with the enumeration of planar triangulations [4]. To our knowledge, the generic case remains open. If we consider instead the reverse collection of steps (Proposition 14), then it is the number of walks ending at $(i, i)$ that is remarkably simple. This is a new result, which we would like to see explained in a more combinatorial manner.

- Motzkin numbers: this famous sequence of numbers arises in the solution of the cases $\mathcal{S}=\{\bar{x}, y, x \bar{y}\}$ and $\mathcal{S}=\{x, \bar{x}, y, \bar{y}, x \bar{y}, \bar{x} y\}$ (Propositions 9 and 10). The first problem is equivalent to the enumeration of involutions with no decreasing subsequence of length 4 , and the occurrence of Motzkin numbers follows from restricting a bijection of Françon and Viennot 24. The solution to the second problem is, to our knowledge, new, and deserves a more combinatorial solution. Can one find a direct explanation for why the respective counting sequences for the total number of walks of these two models differ by a power of 2 ? Is there a connection with the $2^{n}$ phenomenon of [19]?

- Gessel's walks: although we have not solved this case $(\mathcal{S}=\{x, \bar{x}, x y, \bar{x} \bar{y}\})$ in this paper, we cannot resist advertising Gessel's former conjecture, which has now become Kauers-Koutschan-Zeilberger's theorem:

$$
q(0,0 ; 2 n)=16^{n} \frac{(5 / 6)_{n}(1 / 2)_{n}}{(5 / 3)_{n}(2)_{n}} .
$$

Certain other closed form expressions obtained in this paper are less mysterious. As discussed at the beginning of Section 5.3, walks with E, W, NW and SE steps are in bijection with pairs of non-intersecting walks. The Gessel-Viennot method (which, as our approach, is an inclusionexclusion argument) expresses the number of walks ending at $(i, j)$ as a 2-by-2 determinant, thus justifying the closed form expressions of Proposition 11. The extension of this theory by Stembridge [53 allows one to let $i$, or $j$, or both $i$ and $j$ vary, and the number of walks is now expressed as a pfaffian. Hence the closed forms of Corollary 12 are not unexpected. However, one may try to find direct proofs not involving the inclusion-exclusion principle. Moreover, the following question may be interesting per se:

- Walks with E, W, NW and SE steps: can one explain bijectively why as many $2 m$-step walks end on the $x$ - and $y$-axes? Recall that those ending on the $x$-axis were counted bijectively in [29].

Another well-understood case is that of quarter plane walks with N, E, S and W steps. The number of such walks ending at the origin is a product of Catalan numbers, and this has been explained bijectively, first in a recursive manner [18], and more recently directly, using again certain planar maps as intermediate objects [5]. Another argument, based on the reflection principle and thus involving minus signs, appears in [31] and applies to more general endpoints. 


\subsection{Explain algebraic series}

A related problem is to explain combinatorially, via a direct construction, why the three models of Section 6 have algebraic generating functions. Given the connection between Kreweras' walks and planar triangulations [4], this could be of the same complexity as proving directly that families of planar maps have an algebraic generating function. (Much progress has been made recently on this problem by Schaeffer, Di Francesco and their co-authors.) And what about Gessel's walks (with steps E, W, NE and SW), which we have not solved in this paper, but have very recently been proved to have an algebraic generating function as well [9]?

\subsection{Models with a vertical symmetry}

When $\mathcal{S}$ is invariant by a reflection across a vertical axis, the group $G(\mathcal{S})$ has cardinality 4 and Proposition 8 gives the generating function $Q(x, y ; t)$ as the positive part of a rational function. We have not worked out the coefficient extraction in any of these 16 cases. This may be worth doing, with the hope of finding closed form expressions in some cases. However, according to [8], there is little hope to find an algebraic solution for $Q(1,1 ; t)$.

\subsection{Models with an infinite group}

Two of the 56 models that are associated with an infinite group (Table 1 ) have been proved to have a non-D-finite generating function 41]. We conjecture that this holds for all models with an infinite group. This conjecture is based on our experimental attempts to discover a differential equation satisfied by the generating function, and much strengthened by the further attempts of Bostan and Kauers [8], which are based on the calculation of 1000 terms of each generating function. It also relies on the fact that all equations with two catalytic variables and an infinite group that have been solved so far have a non-D-finite solution. How could one prove this conjecture, for instance in the case $\mathcal{S}=\{\mathrm{N}, \mathrm{E}, \mathrm{NE}, \mathrm{SW}\}$ ? And what is the importance, if any, that can be attributed to the fact that the step sets with finite groups either exhibit a symmetry across the $y$-axis or have a vector sum of zero?

\subsection{Automatic derivation of differential equations}

Our D-finite but transcendental solutions are expressed as the positive part (in $x$ and $y$ ) of a 3-variable rational series in $t, x$ and $y$ (Proposition 8). Can one derive automatically from these expressions differential equations satisfied by $Q(0,0 ; t)$ and $Q(1,1 ; t)$ ? This would be a convenient way to fill in the gap between our work and the paper [8], where differential equations are conjectured for the series $Q(1,1 ; t)$.

For the algebraic solutions of Section 6 , it is easy to derive from our results first an algebraic equation satisfied by $Q(0,0 ; t)$ (or $Q(1,1 ; t)$ ), and then a differential equation satisfied by this series, using the MAPLE package GFun [50].

\subsection{Variations and extensions}

It is natural to ask to which similar problems the approach used in this paper could be adapted. To make this question more precise, let us underline that such problems may involve, for instance, putting weights on the walks, allowing more general steps, or considering higher dimensions. However, the very first question is whether Gessel's model can be dealt with using the ideas of this paper!

Weighted paths. One may try to adapt our approach to solve refined enumeration problems. For instance, some authors have studied the enumeration of walks in a wedge, keeping track not only of the number of steps, but also of the number of contacts, or visits to the boundary lines [32, 44, 43]. Of course, other statistics could be considered.

Another natural way to add weights, of a more probabilistic nature, consists in studying Markov chains confined to the quarter plane. The weight of a walk is then its probability. An entire book is devoted to the determination of the stationary distributions of such chains [21]. 
These distributions are governed by functional equations similar to ours, but without the length variable $t$ (since only the stationary regime is considered). This difference makes the problem rather different in nature, and indeed, the tools involved in [21] are much more analytic than algebraic. A natural way to set the problem back in the algebraic playground (to which our power series methods belong) is to keep track of the length of the trajectories, which boils down to studying the law of the chain at time $n$. This was done in [13] for a probabilistic version of Kreweras' walks, using a variant of the method presented in this paper. An asymptotic analysis of the solution should then yield the limiting/stationary distribution. This was however not done in [13]. Instead, we enriched our algebraic approach with a few basic analytic arguments to solve directly the equation that describes the stationary distribution. This solution is in our opinion more elementary than the original ones 21, 23]. It is worth noting that the bivariate series that describes the stationary distribution is algebraic, and that this Kreweras chain is actually the main algebraic example of 21. In view in the results presented in this paper, it is natural to ask if one could design probabilistic versions of the other three algebraic models (Propositions 14 and 15, plus Gessel's model) that would also yield algebraic stationary distributions.

Conversely, it is natural to ask whether certain tools from [21], other than the group of the walk, could be adapted to our power series context. We are thinking in particular of the material of Chapter 4, which is devoted to the case where the group of the walk is finite, and (under this hypothesis) to the conditions under which the stationary distribution has an algebraic generating function.

More general steps. The fact that we only allow "small" steps plays a crucial role in our approach, and more precisely in the definition of the group of the walk (Section 3). However, this does not mean that models with larger steps are definitely beyond reach. First, it is always possible to write a functional equation for the series $Q(x, y ; t)$, based on a step-by-step construction of the walk. If no step has a coordinate smaller than -1 , the right-hand side of the equation only involves $Q(0, y ; t)$ and $Q(x, 0 ; t)$, but otherwise more unknown functions, depending exclusively on $x$ or $y$, appear. Another important difference with the present setting is that the kernel has now degree larger than 2 (in $x$ or $y$ ). One can still define a group, but acting on pairs $(x, y)$ than cancel the kernel. We refer to [15] for the solution of a simple example, with steps $(2,-1)$ and $(-1,2)$.

Higher dimension. Finally, a natural question is to address 3-dimensional problems as those studied experimentally in [8]. Provided one focusses on walks with small steps, the key ingredients of our approach - the functional equation and the group of the walk - can indeed be adapted in a straighforward manner to this higher-dimensional context.

\section{Tables}

The tables below list the 79 step sets $\mathcal{S}$ we consider, classified according to the cardinality of the group $G(\mathcal{S})$. The first three tables contain sets for which $G(\mathcal{S})$ has cardinality 4,6 and 8 respectively. The orbit of $(x, y)$ under the action of this group is listed in the second column. The third column lists the steps of $\mathcal{S}$. The fourth one displays the numbers $q(1,1 ; n)$ and $q(0,0 ; n)$ that respectively count all quarter plane walks and quarter plane walks ending at the origin. We have given the reference of these sequences when they appear in the Encyclopedia of Integer Sequences [51. In the rightmost column, we give references on this model, both in this paper and in other papers. 


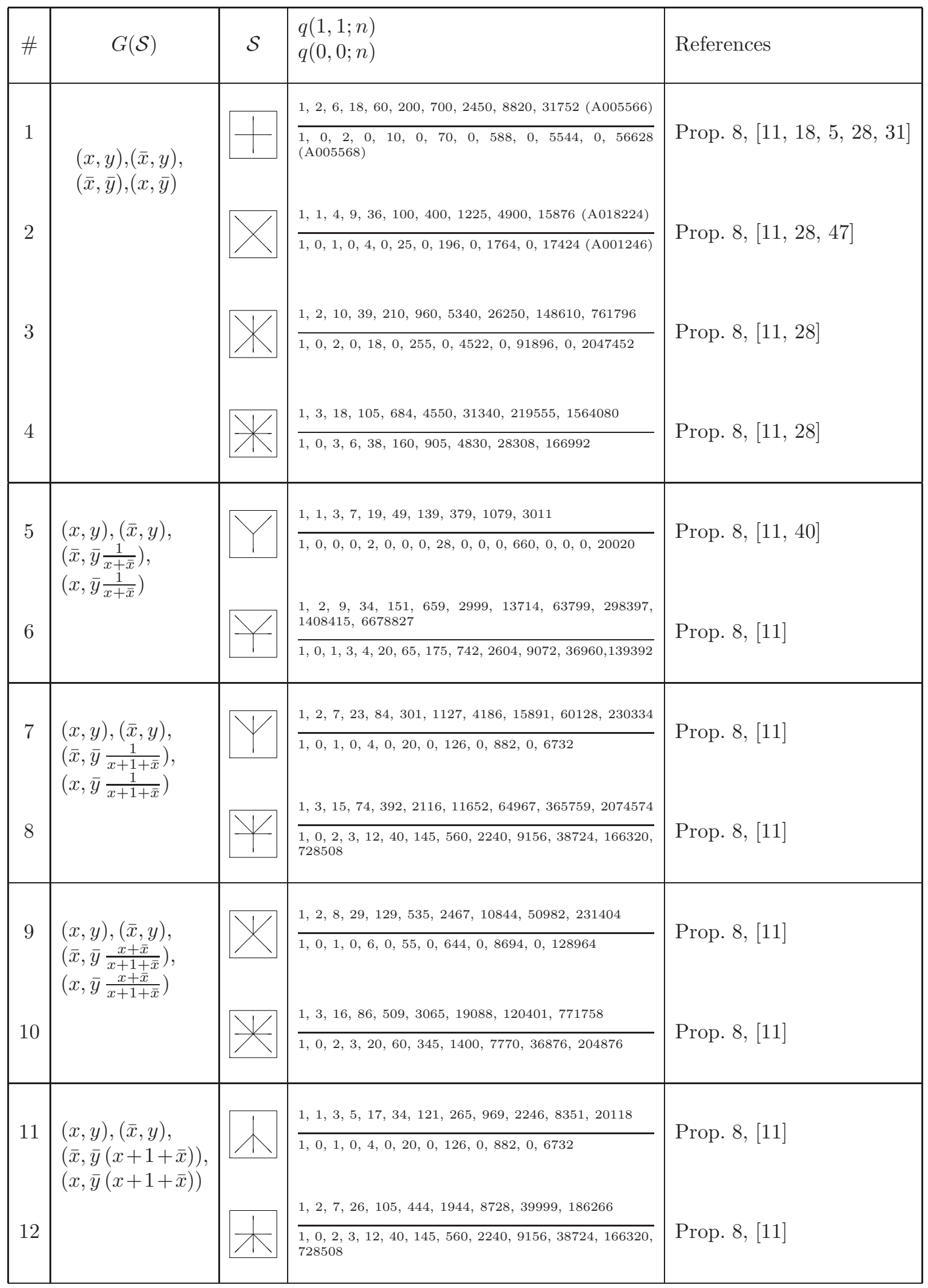




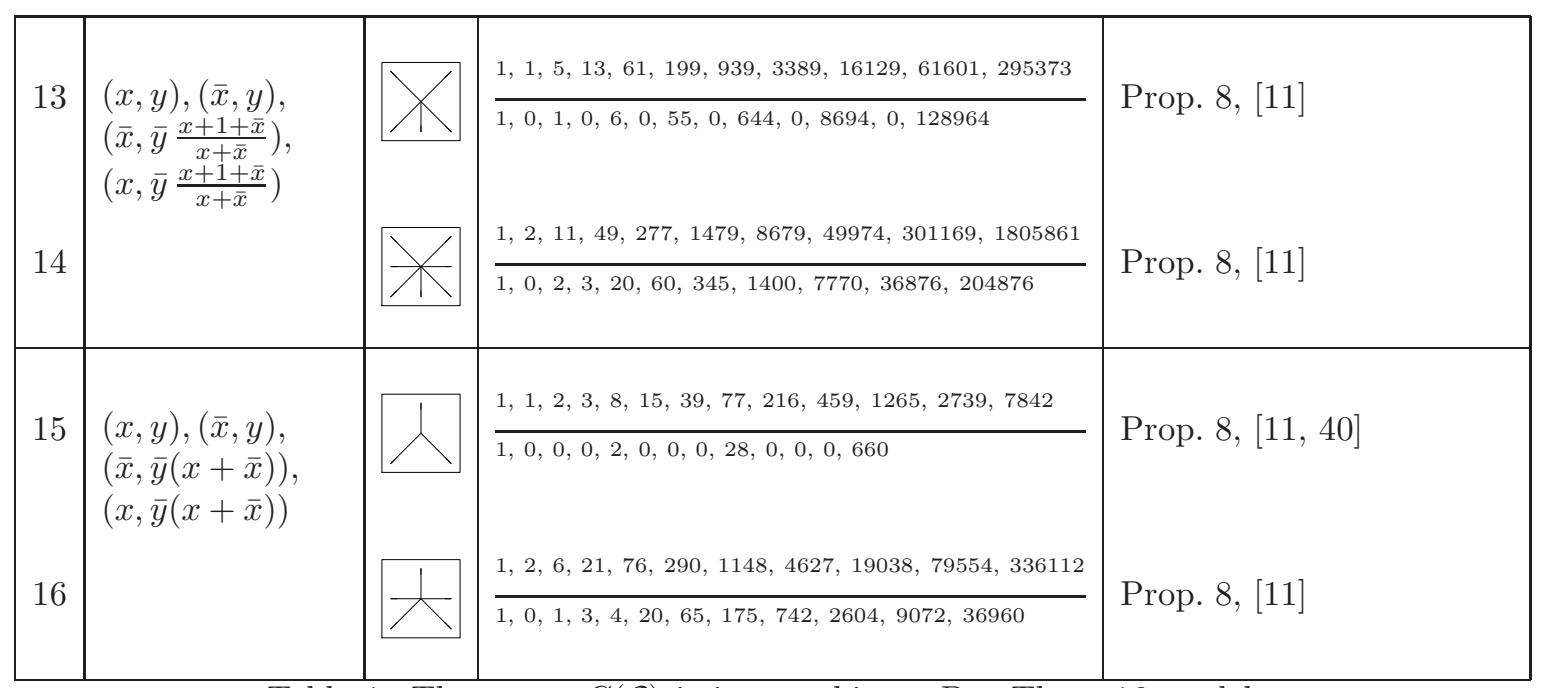

Table 1: The group $G(\mathcal{S})$ is isomorphic to $D_{2}$. These 16 models have a D-finite generating function.

\begin{tabular}{|c|c|c|c|c|}
\hline \# & $G(\mathcal{S})$ & $\mathcal{S}$ & $\begin{array}{l}q(1,1 ; n) \\
q(0,0 ; n)\end{array}$ & References \\
\hline 1 & $\begin{array}{l}(x, y),(\bar{x} y, y) \\
(\bar{x} y, \bar{x}),(\bar{y}, \bar{x}) \\
(\bar{y}, \bar{y} x),(x, \bar{y} x)\end{array}$ & & $\begin{array}{l}\begin{array}{l}1,1,2,4,9,21,51,127,323,835,2188, \\
(\mathrm{~A} 001006)\end{array} \\
\frac{1,0,0,1,0,0,5,0,0,42,0,0,462(\mathrm{~A} 005789)}{} \\
\frac{1,2,8,32,144,672,3264,16256,82688(\mathrm{~A} 129400)}{1,0,2,2,12,30,130,462,1946,7980,34776,153120}\end{array}$ & $\begin{array}{l}\text { Prop. 9, } 28 \\
\text { Prop. 10, 28, }\end{array}$ \\
\hline 3 & $\begin{array}{l}(x, y),(\bar{x} \bar{y}, y) \\
(\bar{x} \bar{y}, x),(y, x), \\
(y, \bar{x} \bar{y}),(x, \bar{x} \bar{y})\end{array}$ & & $\frac{1,1,3,7,17,47,125,333,939,2597,7183}{1,0,0,2,0,0,16,0,0,192,0,0,2816 \text { (A006335) }}$ & Prop. 13, 137, 13, \\
\hline 4 & & & $\frac{1,2,4,10,26,66,178,488,1320,3674,10318}{1,0,0,2,0,0,16,0,0,192,0,0,2816 \text { (A006335) }}$ & Prop. 14, 40, \\
\hline 5 & & $y$ & $\frac{1,3,14,67,342,1790,9580,52035,285990}{1,0,3,4,26,80,387,1596,7518,34656,167310}$ & Prop. 15 \\
\hline
\end{tabular}

Table 2: The group $G(\mathcal{S})$ is isomorphic to $D_{3}$. These five models have a D-finite generating function, and the last three even have an algebraic generating function. 


\begin{tabular}{|c|c|c|c|c|}
\hline$\#$ & $G(\mathcal{S})$ & $\mathcal{S}$ & $\begin{array}{l}q(1,1 ; n) \\
q(0,0 ; n)\end{array}$ & References \\
\hline 1 & $\begin{array}{l}(x, y),(y \bar{x}, y) \\
\left(y \bar{x}, y \bar{x}^{2}\right),\left(\bar{x}, y \bar{x}^{2}\right) \\
(\bar{x}, \bar{y}),(x \bar{y}, \bar{y}) \\
\left(x \bar{y}, x^{2} \bar{y}\right),\left(x, \bar{y} x^{2}\right)\end{array}$ & & $\frac{1,1,3,6,20,50,175,490,1764,5292(\mathrm{~A} 005558)}{1,0,1,0,3,0,14,0,84,0,594,0,4719(\mathrm{~A} 005700)}$ & Prop. 11, 28, 29, 44 \\
\hline 2 & $\begin{array}{l}(x, y),(\bar{x} \bar{y}, y) \\
\left(\bar{x} \bar{y}, x^{2} y\right),\left(\bar{x}, x^{2} y\right) \\
(\bar{x}, \bar{y}),(x y, \bar{y}) \\
\left(x y, \bar{x}^{2} \bar{y}\right),\left(x, \bar{y} \bar{x}^{2}\right)\end{array}$ & & 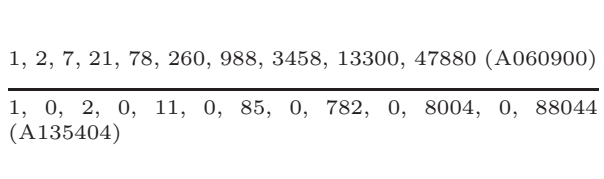 & $35,9,45$ \\
\hline
\end{tabular}

Table 3: The group $G(\mathcal{S})$ is isomorphic to $D_{4}$. Both models have a D-finite generating function, and the second one even has an algebraic generating function.

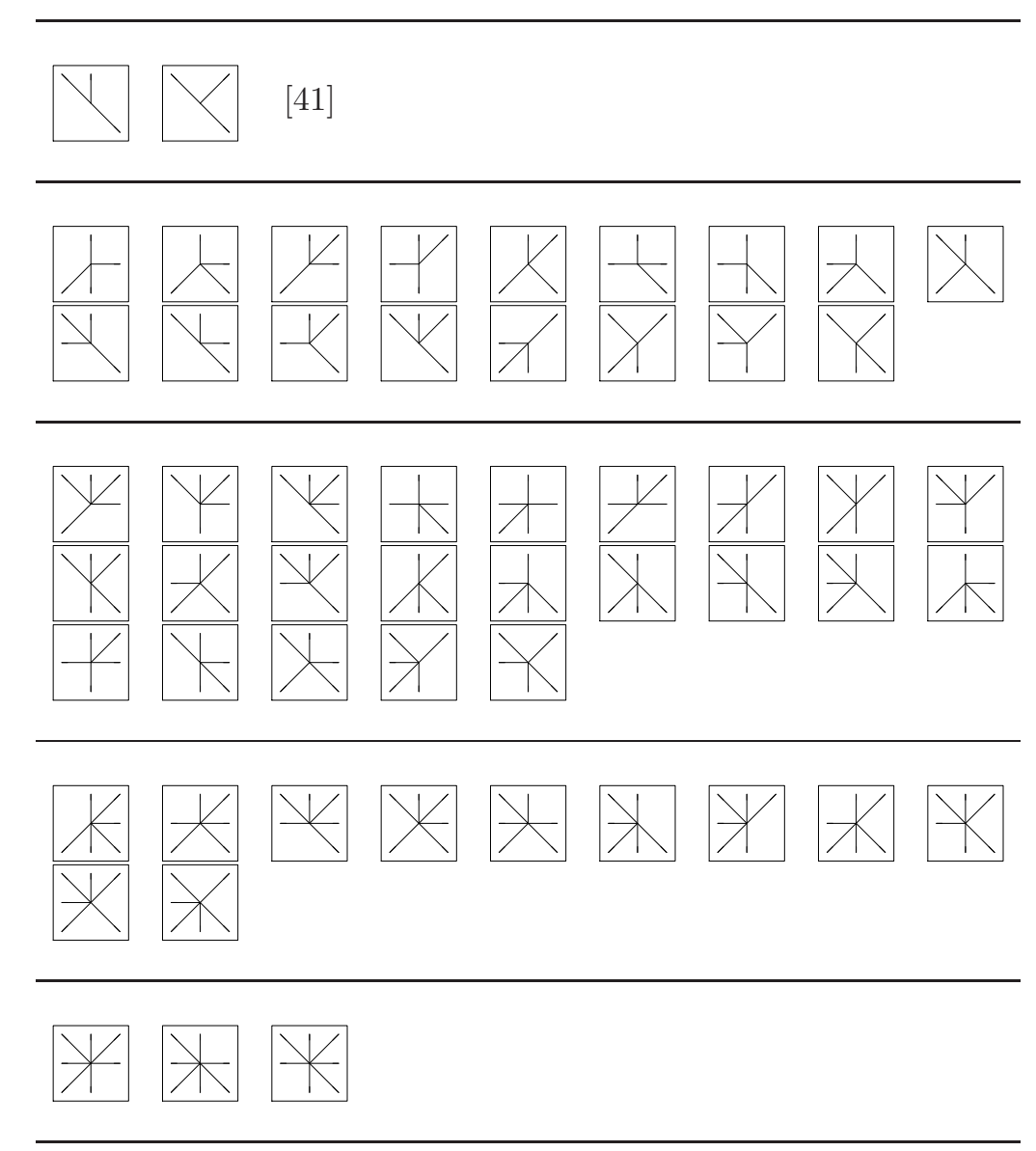

TABLE 4. These 56 step sets are associated with an infinite group. 
Fixed point $\quad$ Condition $\quad \bar{\chi}(X)$

\begin{tabular}{|c|c|c|c|}
\hline \multicolumn{4}{|c|}{ Four steps } \\
\hline t & $\left(a, a^{-2}\right)$ & $a^{4}+a^{3}-1$ & $X^{8}+9 X^{7}+31 X^{6}+62 X^{5}+77 X^{4}+62 X^{3}+31 X^{2}+9 X+1$ \\
\hline$\square$ & $\left(a, \frac{1-a^{2}}{a^{2}}\right)$ & $a^{5}-a^{4}+a^{3}+2 a^{2}-1$ & $\begin{array}{l}X^{10}+18 X^{9}+125 X^{8}+439 X^{7}+897 X^{6}+1131 X^{5}+897 X^{4}+ \\
439 X^{3}+125 X^{2}+18 X+1\end{array}$ \\
\hline$\square$ & $(a, a)$ & $a^{4}+a^{3}-1$ & $X^{8}-19 X^{7}-X^{6}-124 X^{5}+3 X^{4}-124 X^{3}-X^{2}-19 X+1$ \\
\hline$\varnothing$ & $\left(b^{2} / 2, b\right)$ & $b^{6}+b^{4}-4$ & $X^{6}+9 X^{5}+59 X^{4}+70 X^{3}+59 X^{2}+9 X+1$ \\
\hline
\end{tabular}

\begin{tabular}{|c|c|c|c|}
\hline 幽 & $(-1,1)$ & & $2 X^{2}+3 X+2$ \\
\hline F & $\left(a, \frac{a^{2}}{1-a^{2}}\right)$ & $a^{5}+a^{3}+2 a^{2}-1$ & $\begin{array}{l}X^{10}+16 X^{9}+106 X^{8}+371 X^{7}+764 X^{6}+967 X^{5}+764 X^{4}+ \\
371 X^{3}+106 X^{2}+16 X+1\end{array}$ \\
\hline t & $(a, a)$ & $a^{3}-a-1$ & $X^{6}+8 X^{5}+28 X^{4}+41 X^{3}+28 X^{2}+8 X+1$ \\
\hline$甘$ & $\left(a, a^{-2}\right)$ & $a^{3}-a-1$ & $X^{6}+2 X^{5}+6 X^{4}+5 X^{3}+6 X^{2}+2 X+1$ \\
\hline X & $\left(\frac{2 b^{2}}{1-b^{2}}, b\right)$ & $3 b^{6}+b^{4}+b^{2}-1$ & $X^{6}+14 X^{5}+87 X^{4}+100 X^{3}+87 X^{2}+14 X+1$ \\
\hline 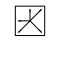 & $(a, 1 / a)$ & $a^{2}+a+1$ & $X^{4}+17 X^{3}+81 X^{2}+17 X+1$ \\
\hline
\end{tabular}

\begin{tabular}{|c|c|c|c|}
\hline 世 & $\left(a, 2+a-3 a^{2}-3 a^{3}\right)$ & $\begin{array}{l}3 a^{6}+6 a^{5}+2 a^{4}-5 a^{3}- \\
4 a^{2}+1\end{array}$ & $\begin{array}{l}X^{12}+23 X^{11}+283 X^{10}+1861 X^{9}+7461 X^{8}+14225 X^{7}+ \\
18249 X^{6}+14225 X^{5}+7461 X^{4}+1861 X^{3}+283 X^{2}+23 X+1\end{array}$ \\
\hline 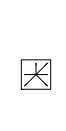 & $\left(\frac{2+2 b-b^{3}}{b^{2}\left(1+b+b^{2}\right)}, b\right)$ & $\begin{array}{l}b^{7}+b^{6}+2 b^{5}+5 b^{4}+ \\
4 b^{3}-4 b^{2}-8 b-4\end{array}$ & $\begin{array}{l}X^{14}+37 X^{13}+567 X^{12}+4853 X^{11}+26197 X^{10}+89695 X^{9}+ \\
194611 X^{8}+250446 X^{7}+194611 X^{6}+89695 X^{5}+26197 X^{4}+ \\
4853 X^{3}+567 X^{2}+37 X+1\end{array}$ \\
\hline « & $(a, a)$ & $a^{4}+a^{3}-1$ & $\begin{array}{l}X^{8}+117 X^{7}+3671 X^{6}+13396 X^{5}+19683 X^{4}+13396 X^{3}+ \\
3671 X^{2}+117 X+1\end{array}$ \\
\hline
\end{tabular}

Seven steps

\begin{tabular}{|c|c|c|c|}
\hline & $(a, 1 / a)$ & $a^{3}-a-1$ & $X^{6}+28 X^{5}+224 X^{4}+345 X^{3}+224 X^{2}+28 X+1$ \\
\hline
\end{tabular}

Table 5: Proving that $G(\mathcal{S})$ is infinite.

Acknowledgements. We are grateful to Michael Albert, Jason Bell, Pierrette Cassou-Nogues, Julie Déserti, Arnaud Jehanne and Andrew Rechnitzer for various discussions and advice related to this paper. 


\section{References}

[1] D. André. Solution directe du problème résolu par M. Bertrand. C. R. Acad. Sci. Paris, 105:436-437, 1887.

[2] C. Banderier and P. Flajolet. Basic analytic combinatorics of directed lattice paths. Theoret. Comput. Sci., 281(1-2):37-80, 2002.

[3] L. Bayle and A. Beauville. Birational involutions of $\mathbf{P}^{2}$. Asian J. Math., 4(1):11-17, 2000.

[4] O. Bernardi. Bijective counting of Kreweras walks and loopless triangulations. J. Combin. Theory Ser. A, 114(5):931-956, 2007.

[5] O. Bernardi. Bijective counting of tree-rooted maps and shuffles of parenthesis systems. Electron. J. Combin., 14(1):Research Paper 9, 36 pp. (electronic), 2007.

[6] J. Bertrand. Solution d'un problème. C. R. Math. Acad. Sci. Paris, 105:369, 1887.

[7] J. Blanc. Finite abelian subgroups of the Cremona group of the plane. C. R. Math. Acad. Sci. Paris, 344(1):21-26, 2007.

[8] A. Bostan and M. Kauers. Automatic classification of restricted lattice walks. In FPSAC'09, Discrete Math. and Theoret. Comput. Sci. Proceedings, pages 203-217, 2009. ArXiv:0811.2899v1.

[9] A. Bostan and M. Kauers. The complete generating function for Gessel walks is algebraic. ArXiv:0909.1965, 2009.

[10] M. Bousquet-Mélou. Walks on the slit plane: other approaches. Adv. in Appl. Math., 27(2-3):243-288, 2001.

[11] M. Bousquet-Mélou. Counting walks in the quarter plane. In Mathematics and computer science 2, (Versailles, 2002), Trends Math., pages 49-67. Birkhäuser, Basel, 2002.

[12] M. Bousquet-Mélou. Four classes of pattern-avoiding permutations under one roof: generating trees with two labels. Electron. J. Combin., 9(2):Research paper 19, 31 pp. (electronic), 2002/03.

[13] M. Bousquet-Mélou. Walks in the quarter plane: Kreweras' algebraic model. Ann. Appl. Probab., 15(2):14511491, 2005.

[14] M. Bousquet-Mélou and M. Petkovšek. Linear recurrences with constant coefficients: the multivariate case. Discrete Math., 225(1-3):51-75, 2000.

[15] M. Bousquet-Mélou and M. Petkovšek. Walks confined in a quadrant are not always D-finite. Theoret. Comput. Sci., 307(2):257-276, 2003.

[16] M. Bousquet-Mélou and G. Schaeffer. Walks on the slit plane. Probab. Theory Related Fields, 124(3):305-344, 2002.

[17] M. Bousquet-Mélou and G. Xin. On partitions avoiding 3-crossings. Sém. Lothar. Combin., 54:Art. B54e, 21 pp. (electronic), 2005/07.

[18] R. Cori, S. Dulucq, and G. Viennot. Shuffle of parenthesis systems and Baxter permutations. J. Combin. Theory Ser. A, 43(1):1-22, 1986.

[19] E. Duchi and R. A. Sulanke. The $2^{n-1}$ factor for multi-dimensional lattice paths with diagonal steps. Sém. Lothar. Combin., 2004. Art. B51c, 16 pp. (electronic).

[20] P. Duchon. On the enumeration and generation of generalized Dyck words. Discrete Math., 225(1-3):121-135, 2000.

[21] G. Fayolle, R. Iasnogorodski, and V. Malyshev. Random walks in the quarter-plane: Algebraic methods, boundary value problems and applications, volume 40 of Applications of Mathematics. Springer-Verlag, Berlin, 1999.

[22] P. Flajolet. Analytic models and ambiguity of context-free languages. Theoret. Comput. Sci., 49(2-3):283309, 1987.

[23] L. Flatto and S. Hahn. Two parallel queues created by arrivals with two demands. I. SIAM J. Appl. Math., 44(5):1041-1053, 1984

[24] J. Françon and G. Viennot. Permutations selon leurs pics, creux, doubles montées et double descentes, nombres d'Euler et nombres de Genocchi. Discrete Math., 28(1):21-35, 1979.

[25] I. M. Gessel. A factorization for formal Laurent series and lattice path enumeration. J. Combin. Theory Ser. A, 28(3):321-337, 1980.

[26] I. M. Gessel. A probabilistic method for lattice path enumeration. J. Statist. Plann. Inference, 14(1):49-58, 1986.

[27] I. M. Gessel and X. Viennot. Binomial determinants, paths, and hook length formulae. Adv. in Math., pages 300-321, 1985.

[28] I. M. Gessel and D. Zeilberger. Random walk in a Weyl chamber. Proc. Amer. Math. Soc., 115(1):27-31, 1992.

[29] D. Gouyou-Beauchamps. Chemins sous-diagonaux et tableaux de Young. In Combinatoire énumérative (Montréal, 1985, Québec), volume 1234 of Lecture Notes in Math., pages 112-125. Springer, Berlin, 1986.

[30] D. Gouyou-Beauchamps. Standard Young tableaux of height 4 and 5. European J. Combin., 10(1):69-82, 1989.

[31] R. K. Guy, C. Krattenthaler, and B. E. Sagan. Lattice paths, reflections, \& dimension-changing bijections. Ars Combin., 34:3-15, 1992.

[32] E. J. Janse van Rensburg. Adsorbing staircase walks and staircase polygons. Ann. Comb., 3(2-4):451-473, 1999. 
[33] E. J. Janse van Rensburg, T. Prellberg, and A. Rechnitzer. Partially directed paths in a wedge. J. Combin. Theory Ser. A, 115(4):623-650, 2008.

[34] S. Kantor. Neue Theorie der eindeutigen periodischen Transformationen in der Ebene. Acta Math., 19(1):115193, 1895.

[35] M. Kauers, C. Koutschan, and D. Zeilberger. Proof of Ira Gessel's lattice path conjecture. Proc. Nat. Acad. Sci. USA, 106(28):11502-11505, 2009. ArXiv:0806.4300.

[36] M. Kauers and D. Zeilberger. The quasi-holonomic Ansatz and restricted lattice walks. J. Difference Equ. Appl., 14:1119-1126, 2008. ArXiv:0806.4318.

[37] G. Kreweras. Sur une classe de problèmes liés au treillis des partitions d'entiers. Cahiers du B.U.R.O., 6:5-105, 1965.

[38] L. Lipshitz. The diagonal of a $D$-finite power series is D-finite. J. Algebra, 113(2):373-378, 1988.

[39] L. Lipshitz. D-finite power series. J. Algebra, 122:353-373, 1989.

[40] M. Mishna. Classifying lattice walks restricted to the quarter plane. J. Combin. Theory Ser. A, 116(2):460477, 2009. ArXiv:math/0611651.

[41] M. Mishna and A. Rechnitzer. Two non-holonomic lattice walks in the quarter plane. Theoret. Comput. Sci., 410(38-40):3616-3630, 2009. ArXiv:math/0701800.

[42] H. Niederhausen. The ballot problem with three candidates. European J. Combin., 4(2):161-167, 1983.

[43] H. Niederhausen. A note on the enumeration of diffusion walks in the first octant by their number of contacts with the diagonal. J. Integer Seq., 8(4):Article 05.4.3, 7 pp. (electronic), 2005.

[44] H. Niederhausen. Random walks in octants, and related structures. J. Statist. Plann. Inference, 135(1):165196, 2005.

[45] M. Petkovsek and H. S. Wilf. On a conjecture of Ira Gessel. ArXiv:0807.3202v1, 2008.

[46] M. Petkovšek, H. S. Wilf, and D. Zeilberger. $A=B$. A K Peters Ltd., Wellesley, MA, 1996.

[47] D. Poulalhon and G. Schaeffer. Applied Combinatorics on Words, volume 105 of Encyclopedia of Mathematics and its Applications, chapter "Coding, counting and sampling with words". Cambridge University Press, 2005.

[48] A. Regev. Asymptotic values for degrees associated with strips of Young diagrams. Adv. in Math., 41(2):115$136,1981$.

[49] B. E. Sagan. The symmetric group. Representations, combinatorial algorithms, and symmetric functions, volume 203 of Graduate Texts in Mathematics. Springer-Verlag, New York, second edition, 2001.

[50] B. Salvy and P. Zimmermann. Gfun: a Maple package for the manipulation of generating and holonomic functions in one variable. ACM Transactions on Mathematical Software, 20(2):163-177, 1994. Reprint doi:10.1145/178365.178368.

[51] N. J. A. Sloane and S. Plouffe. The encyclopedia of integer sequences. Academic Press Inc., San Diego, CA, 1995. http://www.research.att.com/ njas/sequences/index.html.

[52] R. P. Stanley. Enumerative combinatorics 2, volume 62 of Cambridge Studies in Advanced Mathematics. Cambridge University Press, Cambridge, 1999.

[53] J. R. Stembridge. Nonintersecting paths, pfaffians, and plane partitions. Adv. in Math., 83(1):96-131, 1990. Available at http://hdl.handle.net/2027.42/28397.

[54] A. Wiman. Zur Theorie der endlichen Gruppen von birationalen Transformationen in der Ebene. Math. Ann., 48(1-2):195-240, 1896.

[55] G. Xin and T. Y. J. Zhang. Enumeration of bilaterally symmetric 3-noncrossing partitions. Discrete Math., 309(8):2497-2509, 2009. ArXiv:0810.1344.

[56] D. Zeilberger. The umbral transfer-matrix method: I. Foundations. J. Comb. Theory, Ser. A, 91:451-463, 2000.

MBM: CNRS, LaBRI, Université Bordeaux 1, 351 Cours de la Libération, 33405 Talence Cedex, FRANCE

E-mail address: mireille.bousquet@labri.fr

MM: Dept. Mathematics, Simon Fraser University, 8888 University Drive, Burnaby, Canada

E-mail address: mmishna@sfu.ca 